\title{
PROJECTIVE-TYPE DIFFERENTIAL INVARIANTS AND GEOMETRIC CURVE EVOLUTIONS OF KDV-TYPE IN FLAT HOMOGENEOUS MANIFOLDS
}

\author{
G. MARÍ BEFFA
}

\begin{abstract}
In this paper we describe moving frames and differential invariants for curves in two different $|1|$-graded parabolic manifolds $G / H, G=$ $O(p+1, q+1)$ and $G=O(2 m, 2 m)$, and we define differential invariants of projective-type. We then show that, in the first case, there are geometric flows in $G / H$ inducing equations of KdV-type in the projective-type differential invariants when proper initial conditions are chosen. We also show that geometric Poisson brackets in the space of differential invariants of curves in $G / H$ can be reduced to the submanifold of invariants of projective-type to become Hamiltonian structures of KdV-type. The study is based on the use Fels and Olver moving frames. In the second case we classify differential invariants and we show that for some choices of moving frames we can find geometric evolutions inducing a decoupled system of $\mathrm{KdV}$ equations on the projectivetype differential invariants, if proper initial values are chosen. We describe the differences between this case and the Lagrangian Grassmannian case in detail.
\end{abstract}

\section{INTRODUCTION}

A new concept of moving frame has recently been introduced by Fels and Olver in [FO1, FO2]. Instead of the classical concept of moving frame along a curve $u: I \rightarrow M$ as a curve in the frame bundle of $M$ over $u$, Fels and Olver introduced the idea of a moving frame as an equivariant map. For them, a (left-invariant) moving frame of $m$-order along $u$ is a map from the space of jets $J^{(m)}(\mathbb{R}, M)$ to the group $G$, equivariant with respect to the prolonged action of the group $G$ on $J^{(m)}(\mathbb{R}, M)$ and the left action of $G$ on itself. Hints of this idea can be found in the work of Cartan $[\mathrm{C}]$ (who defined a moving frame along a curve in projective space as a matrix in $S L(3)$ ), Green ([G]), Griffiths ([Gr]) and others. In [FO1, FO2] the authors give a constructive method to find moving frames using constant transverse sections to the prolonged leaves of the group (also called normalization equations).

A fundamental problem with the traditional moving frame concept was that in non-affine geometries (projective, conformal, etc) the classical Frenet equation of the moving frame did not provide a complete set of generators of differential invariants for the curve. For example, in the projective case the traditional moving frame method could not be used at all to find projective curvatures. In the conformal case all but two invariants can be found using traditional Frenet equations, the other two invariants need to be found by other means (see [Fi]). This is not a problem

Date: September, 2007.

1991 Mathematics Subject Classification. Primary: 37K; Secondary: 53A55.

Key words and phrases. Invariant evolutions of curves, flat homogeneous spaces, Poisson brackets, differential invariants, projective invariants, completely integrable PDEs, moving frames. 
with Fels and Olver's frame, if $M=G / H$ is a homogeneous space, a complete set of generators of differential invariants can always be found among the entries of the matrix defining its Frenet equation $([\mathrm{H}])$.

The reason for the shortcomings of the traditional approach was explained in [M3] when $M=G / H$ is a homogeneous space. Indeed, if $\rho: J^{(k)}(\mathbb{R}, M) \rightarrow G$ is a left invariant moving frame and $\phi_{\rho}: M \rightarrow M$ is defined as the action of $\rho\left(u^{(k)}\right)$ on $M$, that is, $\phi_{\rho}(u)=\rho\left(u^{(k)}\right) \cdot u$, then $d \phi_{\rho}(o)$, identified with an element of $G L(n, \mathbb{R})$, contains in columns a classical moving frame along the curve, where $o=[H] \in G / H$. That is, only invariants determined by the linear action of the group can be determined using the classical moving frame. One needs to consider a moving frame that will take into account the complete action to ensure that all invariants will be found. The authors of [FO2] pointed out that equivariant moving frames can be interpreted in terms of higher order frame bundles. From that point of view, classical frames involve only first order frames.

This paper studies the geometry of curves $u: I \rightarrow M=G / H, G / H$ flat, for two different cases where $G$ is semisimple and its Lie algebra has a length one gradation (these are called $|1|$-graded parabolic manifolds). We study the cases $G=O(p+$ $1, q+1)$ and $G=O(2 m, 2 m)$. We want to find and classify all differential invariants for these curves and study the evolution under some geometric flows of what we will call differential invariants of projective type. We will consider $G$-invariant evolution of curves in $G / H$, or geometric evolutions. If a curve evolution in $G / H$ is invariant under the group, it induces an evolution on the differential invariants of the flow. We aim to identify completely integrable systems that are related to geometric evolutions much like the nonlinear Shrödinger equation is related to the Vortex filament flow via the Hasimoto transformation ([Ha], see also [Ca]). These are called geometric realizations of the integrable system.

Some examples have already been studied by the author, namely that of the Möbius sphere, or local model for flat conformal manifolds ([M2], the choice is $G=$ $O(n+1,1))$, the case of flat projective $\mathbb{R P}^{n}([\mathrm{M} 3]$, with choice $G=P S L(n+1, \mathbb{R})$ ), and the case of the Lagrangian Grassmannian or manifold of Lagrangian planes in $\mathbb{R}^{2 n}$ ([M4], with choice $G=\operatorname{Sp}(2 n)$ ). In the conformal case there exist two projective type differential invariants. Furthermore, one can find a geometric evolution of curves such that a level set of the evolution is given by a complexly coupled system of $\mathrm{KdV}$ equations on these differential invariants. In the projective case all invariants are projective-type, and one can find a geometric evolution of curves in $\mathbb{R P}^{n}$ inducing an Adler-Gel'fand-Dikii evolution on the projective invariants of the flow. In the case of the Lagrangian Grassmannian there exist a geometric evolution of curves inducing an evolution on its differential invariants that has as level set a system of decoupled KdV equations on the differential invariants of projective type. The author has conjectured that these are the only types of KdV evolutions appearing in $|1|$-graded parabolic manifolds.

A paper by Kobayashi $([\mathrm{KO}])$ classifies all $|1|$-graded parabolic manifolds. Indeed, for those manifolds the Lie algebra $\mathfrak{g}$ is a direct sum of the simple Lie algebras listed in $[\mathrm{KO}]$ and the group actions decouple. Hence, it suffices to study each one of the cases listed in [KO] (we will not work on the exceptional cases). This paper studies the cases $G=O(p+1, q+1)$ and $G=O(2 m, 2 m)$, while a projective case, $S L(p+q)$, and $O(2 m+1,2 m+1)$ (possibly similar to $O(2 m, 2 m)$ ) will be left for later papers. 
One of the main difficulties of these studies, if not the main one, is that moving frames along curves for homogenous manifolds are not known in general, only in some cases. In particular they were not known for the cases presented here and the ones that are still pending. In fact, to be able to effectively study the Poisson brackets for PDEs associated to these (Cartan) geometries one needs not only to find the moving frames, but to find moving frames of a convenient enough form so that the evolution of the differential invariants under geometric flows is clear and recognizable as completely integrable systems. The identification of the associated integrable evolutions is usually based on the identification of Hamiltonian structures that are obtained by reduction to the space of differential invariants (the so-called geometric Poisson brackets for PDEs). Hence, identifying the result of such reduction requires that the space of differential invariants is not only described, but described in a form that is simple enough for further use. In the case of $G=S L(p+q)$ such a frame requires a different approach from the one presented in this paper, while in the case of $O(2 m+1,2 m+1)$ the quotient can be identified with skew-symmetric matrices. The evolving one dimensional kernel of generic $2 m+1$ dimensional skew symmetric matrices complicates the calculation of the moving frame with the techniques used here. Even without this problem, one can already see complications in the $O(2 m, 2 m)$ case. Indeed, we prove that no choice of constant transverse section (or zero order in the differential invariants of projective type) will result in a geometric evolution preserving vanishing fifth order differential invariants and inducing $\mathrm{KdV}$-type evolutions on the invariants of projective type. This is the first example of $|1|$-graded parabolic manifolds for which this happens for any constant section of the prolonged leaves. On the other hand, one can find geometric evolutions that preserve the submanifold of vanishing fourth order differential invariants, but not the fifth order ones. In fact, for most dimensions a moving frame can be found via a constant cross section such that on the submanifold of vanishing fourth order differential invariants the evolutions of projective-type and fifth order invariants decoupled. The first group evolves following a decoupled system of $\mathrm{KdV}$ equations (like the Lagrangian Grassmannian case). It will be very interesting to study the geometry of these manifolds and to learn what factors determine a proper choice of invariants.

The reader will notice similarities between the two cases we study in this paper. It is perhaps possible to write a common description of the moving frame calculation up to a certain point. But because soon the results become different and need different treatment we have opted for individual explanations, even if some repetition takes place. Some calculations are similar to those in [M2] and [M4] and we will refer the reader to those papers when such is the case.

In section 2 we describe Fels and Olver definition of moving frame and their method to find them. We also list the results that will be needed along the paper. This method is used in sections 4.1 and 5.1 to find the moving frames in the cases of $G=O(p+1, q+1)$ and $G=O(2 m, 2 m)$, respectively. The moving frames are then used to classify differential invariants and Frenet equations for these two cases. The three sections can be read independently from the rest of the paper.

In section 3 we describe Geometric Hamiltonian structures and their properties. We also describe how these brackets are directly related to some evolutions of curves in $G / H$ for any $|1|$-graded parabolic manifold, that is, one can always find geometric realizations of the Hamiltonian evolutions in $G / H$. We use these definitions and 
the results listed in section 3 to obtain biHamiltonian evolutions of KdV type for projective-type differential invariants in the case of $G=O(p+1, q+1)$. We show that the situation is very much like the situation in the conformal case. These evolutions have a geometric realization on $G / H$ that we give explicitly, together with its biHamiltonian structures. This is described in 4.2. The case $G=O(2 m, 2 m)$ is studied in 5.2. There we show that no choice of constant or zero order transverse section generating the moving frame can preserve the manifold of vanishing fifth order invariants. Furthermore, we show that for most dimensions the evolutions of third and fifth order decouple and the third order follows a decoupled system of KdV equations. We explain the basic differences between this case and the Lagrangian Grassmannian case, the main one being the appearance in the $O(2 m, 2 m)$ case of differential invariants of fifth and higher order that did not exist in the former.

\section{MOVing FRAmes}

In this section we will briefly describe the method of moving frames as redesigned by Fels and Olver in [FO1] and [FO2], and adapted to our particular case.

Definition 1. Let $G$ be a Lie group acting on a manifold $M$. We say that $G$ acts effectively on subsets if, for every open subset $U \subset M$, the global isotropic subgroup of $U$ given by

$$
G_{U}=\{g \in G \text { such that } g \cdot s=s, \text { for all } s \in U\}
$$

is trivial, that is, equal to $\{e\}$. We say $\mathrm{G}$ acts locally effective in subsets if $G_{U}$ is a discrete subgroup of $G$ for every open set $U \subset M$. We say the group acts freely on $M$ if the isotropy subgroups $G_{x}=\{g \in G$ such that $g \cdot x=x\}$ are all trivial.

Definition 2. Let $G$ be an $r$-dimensional Lie group acting on an $n$-dimensional manifold. We say that the group acts regularly on $M$ if all its orbits have the same dimension and each point $x \in M$ has arbitrarily small neighborhoods whose intersection with each orbit is a connected subset thereof. If the second condition in the definition is omitted we say the group acts semi-regularly.

Definition 3. Given a transformation group $G$ acting on a manifold $M$, a moving frame is a smooth $G$-equivariant map

$$
\rho: M \rightarrow G \text {. }
$$

Since we can consider either the right or the left action of $G$ on itself, we can talk about right or left moving frames. The following theorem determines which group actions admit a moving frame. Its proof can be found in [FO1].

Theorem 1. If $G$ acts on $M$, then a moving frame exists in a neighborhood of a point $x \in M$ if, and only if $G$ acts freely and regularly near $x$.

Perhaps the most interesting part of Fels and Olver's method is that it describes a simple way of constructing moving frames based on the so-called normalization equations. One can also find a generating set of differential invariants using those equations.

Definition 4. Assume $G$ acts semi-regularly on $M$ with orbits of dimension $s$. A local cross-section to the orbits is an $r$-dimensional submanifold, $C, r=\operatorname{dim}(M)-s$, such that $C$ intersects each orbit transversally. If $C$ intersects each orbit in one point at most, then we say $C$ is a regular cross-section. 
Definition 5. Let $C \subset M$ be a local cross-section to the $G$ orbits. The normalization equations associated with $C$ are the system of equations

$$
w=g \cdot z=c
$$

where $c \in C$.

It is quite simple to see that, if $G$ acts freely and $C$ is a regular cross section, then there is a unique solution $g=\rho(z)$ to the normalization equations, determining the right moving frame associated with $C$. Moving frames are used to find a complete set of invariants of the action. These invariants can be found in two different ways, the first one being described by the following Theorem.

Theorem 2. If $\rho(x)$ is a (right invariant) moving frame, then the components of the map $I: M \rightarrow M$ defined by $I(x)=\rho(x) \cdot x$ provide a complete set of invariants for the group.

Therefore, the normalization procedure provides a simple direct method for determining invariants of free group actions. Next, we will describe the situation when the manifold $M$ is the jet space associated to curves in a homogeneous space. A variation of what will be defined below needs to be adopted whenever the group $G$ acts also on the independent variable, which is not the case at hand.

Definition 6. Given a manifold $M$, we define the $n$th order jet bundle $J^{(n)}(\mathbb{R}, M)$ to be the set of equivalence classes of curves under the equivalence relation of $n$th order contact. The fibers of $\pi^{n}: J^{(n)}(\mathbb{R}, M) \rightarrow M$ are generalized Grassman manifolds (see $[\mathrm{O}]$.)

We introduce local coordinates $u=\left(u^{\alpha}\right)$ on $M$. The induced coordinates in the jet bundle $J^{(n)}(\mathbb{R}, M)$ are denoted by $\left(x, u^{(n)}\right)$, where $x$ is the independent variable and where the components of $u^{(n)}$ are $u_{k}^{\alpha}$ representing the $k$ th derivative of the dependent variable $u^{\alpha}$ with respect to $x$, for any $k \leq n$. Any transformation group $G$ acting on $M$ preserves the order of contact between curves. Therefore, there is an induced action of $G$ on the $n$th order jet bundle $J^{(n)}(\mathbb{R}, M)$ known as the $n$th prolongation of $G$. In our special case the prolonged action is locally given by

$$
g \cdot\left(x, u, u_{1}, \ldots, u_{n}\right)=\left(x, g \cdot u,(g \cdot u)_{1}, \ldots,(g \cdot u)_{n}\right)
$$

where, again, the subindex indicates the number of derivatives with respect to $x$. The expression $(g \cdot u)_{k}$ defines a formula in terms of $u, u_{1}, \ldots, u_{k}$ which indeed defines the prolonged action. We are using $(g \cdot u)_{k}$ to represent that formula. The invariants of the prolonged action are naturally called differential invariants. The best known differential invariants are the curvature and torsion for curves in Euclidean space $\left(O(n) \ltimes \mathbb{R}^{n}\right) / O(n) \cong \mathbb{R}^{n}$.

Definition 7. A (nth order) differential invariant is a (locally defined) scalar function $I: J^{(n)}(\mathbb{R}, M) \rightarrow \mathbb{R}$ which is invariant under the prolonged action of $G$.

The key element of Fels and Olver's method is that, for a high enough order of contact, a moving frame always exists for the prolonged action. In fact, a theorem by Ovsiannikov [Ov] (corrected by Olver in [O2]) states that if a group acts (locally) effectively on subsets, then there exists an integer $k_{0}$ such that the prolonged action of the given group is locally free on an open and dense subset of the $k_{0}$ th order jet space. This subset is formed by the so-called regular jets (see [FO1]). Direct application of Theorem 1 implies that, if the group acts (locally) effectively on subsets, 
then for $n$ large enough, there exists a moving frame defined on a neigbourhood of regular jets (that is, for generic curves). Moreover, this moving frame can be found solving the normalization equations (2.2) for some choice of transversal section.

Fels and Olver's method provides also a complete description of generating differential invariants. Let $\rho\left(u^{(k)}\right)$ be a right invariant moving frame. From Theorem $2 \mathrm{a}$ complete set of differential invariants is contained in $\rho\left(u^{(k)}\right) \cdot u_{r}, r=0,1,2, \ldots$ But given the normalization equations, some of these differential invariants are constant. We call the constant invariants phantom differential invariants. If $\rho\left(u^{(k)}\right) \cdot u_{r}=$ $c_{r} \in \mathbb{R}^{n}$, we call $c_{r}$ the $r$-normalization constant. The following theorem can be found in [FO2]. We have simplified and adapted the notation. Although this version applies to our particular group action, it does not cover the general case. For complete information see [?].

Theorem 3. A generating system of differential invariants consist of

(a) All non-phantom zeroth order invariants $\rho \cdot u$ and $x$.

(b) All non-phantom differential invariants $\left(\rho\left(u^{(k)}\right) \cdot u_{r}\right)^{\alpha}$ for which $\left(\rho\left(u^{(k)}\right)\right.$. $\left.u_{r-1}\right)^{\alpha}$ was phantom. (The expression ()$^{\alpha}$ represents the $\alpha$-entry of a vector in $\mathbb{R}^{n}$.)

From this theorem, if $\rho\left(u^{(k)}\right) \cdot u_{r}=c_{r}$ has maximal rank, then all $r$ th order differential invariants will be phantom invariants and differential invariants will have higher order. It is important to point out at this moment that, although not explicit in [FO1, FO2], one has choices other than a constant cross section. Indeed, the normalization constants $c_{r}$ can be chosen to be functions of differential invariants of order lower than $r$ (and their derivatives). The resulting moving frame will still generate all differential invariants (see $[\mathrm{H}]$ ).

A different way to find a complete set of generators of differential invariants is to find the so called Serret-Frenet equations of a moving frame.

Definition 8. Let $\rho: J^{(k)}(\mathbb{R}, G / H) \rightarrow G$ be a (left or right invariant) moving frame. We let $\rho^{*} \omega$ denote the horizontal component of the pull back of the (left) Maurer-Cartan forms. We call $\rho^{*} \omega$ the Serret-Frenet equations associated to $\rho$, and they define the system of linear equations that has $\rho$ as solution.

It is trivial to see that the left invariant Serret-Frenet equations are the negative of the right invariant ones, since the left invariant moving frame is the inverse of the right invariant one. The entries of the Serret-Frenet equations always contain a complete set of generating differential invariants. This was shown in $[\mathrm{H}]$ in general, and it is a consequence of [FO1, FO2] for the case at hand.

\section{Geometric Hamiltonian structures.}

Given a semisimple Lie group $G$ there exist natural families of Poisson brackets defined on the space of loops on the dual of its Lie algebra, $\mathcal{L} \mathfrak{g}^{*}$. They can be defined as follows: let $\mathcal{H}: \mathcal{L g}^{*} \rightarrow \mathbb{R}$ be a functional defined on $\mathcal{L}^{*}$ and let $\frac{\delta \mathcal{H}}{\delta M}(M) \in \mathcal{L} \mathfrak{g}$ be its variational derivative.

Given two functionals on $\mathcal{L}^{*}$ we can define their Kac-Moody Lie-Poisson bracket as the bracket given by the relation

$$
\{\mathcal{H}, \mathcal{G}\}(L)=\left\langle\frac{\delta \mathcal{G}}{\delta M}(M),\left(\frac{\delta \mathcal{H}}{\delta M}(M)\right)_{x}+\mathrm{a} d^{*}\left(\frac{\delta \mathcal{H}}{\delta M}(M)\right)(L)\right\rangle
$$


where here we are identifying $\mathfrak{g}$ and $\mathfrak{g}^{*}$ using the nondegenerate Killing form. This bracket is well-known to be a Poisson bracket in the space of functionals on $\mathcal{L}^{*}$. Furthermore, its symplectic leaves (the leaves where Hamiltonian flows lie) coincide with the orbits in $\mathcal{L}^{*}$ under a Kac-Moody action of the group $\mathcal{L} G$ on $\mathcal{L} \mathfrak{g}^{*}$. This action is given by

$$
A(g)(L)=g^{-1} g_{x}+\mathrm{Ad}^{*}(g) L .
$$

There is an additional family of simpler Poisson brackets defined on $\mathcal{L}^{*}$. Given two functionals on $\mathcal{L} \mathfrak{g}^{*}$ we define their Poisson bracket by the formula

$$
\{\mathcal{H}, \mathcal{G}\}_{0}(k)=\left\langle\frac{\delta \mathcal{G}}{\delta M}(M), \mathrm{ad}^{*}\left(L_{0}\right)\left(\frac{\delta \mathcal{H}}{\delta M}(M)\right)\right\rangle
$$

where $L_{0} \in \mathfrak{g}^{*}$ is any constant element.

These families of Poisson brackets are all known to be compatible, that is, any linear combination of these brackets is also a Poisson bracket.

The definition of geometric Poisson brackets is based on the following fact: one can describe the set of Serret-Frenet equations (or moving coframes) as a quotient of a submanifold of $\mathcal{L} \mathfrak{g}^{*}$ by the Kac-Moody action (3.2) of a properly chosen isotropy subgroup of $\mathcal{L} G$. This implies that the bracket (3.1) can be reduced to the space of invariants and one can easily check that the brackets (3.3) are also reducible. We call these reductions geometric Poisson brackets.

Geometric Poisson brackets are found explicitly as follows. Assume $G$ is semisimple and $G / H$ is flat. It is known ([Oc]) that in that case the Lie algebra has a gradation of the form

$$
\mathfrak{g}=\mathfrak{g}_{-1} \oplus \mathfrak{g}_{0} \oplus \mathfrak{g}_{1}
$$

where $\mathfrak{g}_{1}$ and $\mathfrak{g}_{-1}$ are dual of each other with respect to the Killing form, and where $G_{0} \subset G$, the Lie subgroup associated to $\mathfrak{g}_{0}$, acts, via the adjoint map, linearly on both $\mathfrak{g}_{1}$ and $\mathfrak{g}_{-1}$. The Lie subgroup $G_{0}$ is called the isotropy subgroup of $G$.

First of all, assume that $\rho$ is a left moving frame and $\rho=\rho_{-1} \rho_{0} \rho_{1}$ is the local factorization induced by the splitting (3.4) (locally $G=G_{-1} \cdot G_{0} \cdot G_{1}$ where $G_{i}$ is the subgroup corresponding to $\left.\mathfrak{g}_{i}\right)$. Let $K=\rho^{-1} \rho_{x}=K_{-1}+K_{0}+K_{1}$ be the splitting induced on the associated (left invariant) Serret-Frenet equation. The following theorems can be found in [M1].

Theorem 4. There exists a left moving frame $\rho$ such that $K_{-1}=\Lambda$ is constant and $\rho_{-1}$ can be identified with $u$. $\Lambda$ is determined by the first normalization constants.

Since the adjoint action of $G_{0}$ on $\mathfrak{g}_{-1}$ is linear, any element in $G_{0}$ can be identified with an element in $\operatorname{GL}(n, \mathbb{R})$. The following theorem can be found in [M1].

Theorem 5. Let $\rho$ be a left moving frame and assume $\rho=\rho_{-1} \rho_{0} \rho_{1}$ is as above. If $\rho_{0}$ (acting linearly on $\mathfrak{g}_{-1}$ via the adjoint action) is identified with an element of $\operatorname{GL}(n, \mathbb{R})$, then the columns of $\rho_{0}$ form a classical moving frame along the curve $u$, that is an invariant curve in the frame bundle along the curve.

Let $\mathcal{M} \subset \mathcal{L}^{*}$ be the submanifold generated by loops with values in

$$
\mathfrak{g}_{0} \oplus \mathfrak{g}_{1} \oplus\{\Lambda\}
$$

and with positive $\Lambda$-component. Let $N_{0} \subset G_{0}$ be the isotropy subgroup of $\Lambda$ in $G_{0}$ and let $N=\mathcal{L}\left(G_{1} \cdot N_{0}\right)$. 
Theorem 6. There exists an open set $U \subset \mathcal{M}$ such that $N$ acts on $U$ with action (3.2), and such that $U / N$ can be identified with $\mathcal{K}$, the space of differential invariants. Furthermore, the Poisson bracket (3.1) can be Poisson reduced to $U / N$.

A theorem in [M1] states that the brackets (3.3) can also be easily reduced to $U / N$ whenever $L_{0} \in \mathfrak{g}_{1}$.

There are two significant advantages of reducing (3.1) to $\mathcal{K}$. One of them is that in many cases one can find explicitly the reduced bracket for a choice of $G$. Indeed, writing the explicit geometric bracket becomes an algebraic problem, as we will see later. The other one is that the associated reduced Hamiltonian flow can be readily related to geometric evolutions of curves, that is, evolutions of curves for which the group $G$ takes solutions to solutions. Geometric evolutions are those of the form

$$
u_{t}=F \mathbf{r}=r_{1} F_{1}+r_{2} F_{2}+\cdots+r_{n} F_{n}
$$

where $F=\left(F_{1}, F_{2}, \ldots, F_{n}\right)$ is an invertible matrix, $\left\{F_{i}\right\}$ forms a classical moving frame along $u$ and where $\mathbf{r}=\left(r_{1}, \ldots, r_{n}\right)^{T}$ is a vector whose entries are differential invariants of $u$.

Theorem 7. ([M1]) Let $u(t, x)$ be a family of curves solution of a geometric evolution of the form (3.5). Let $v_{r} \in \mathfrak{g}_{-1}$ be determine by $\mathbf{r}$ if we left-identify $\mathfrak{g}_{-1}$ with the tangent to $G / H$. Let $\mathbf{k}$ be the differential invariants defined by $K=\rho^{-1} \rho_{x}$, where $\rho=\rho_{-1} \rho_{0} \rho_{-1}, \rho_{-1}$ is identified with $u$ and $\rho_{0}$ is determined by $F$ as in Theorem 5. Assume that there exists a local functional $h: \mathcal{K} \rightarrow \mathbb{R}$ and a local extension $\mathcal{H}: \mathcal{M} \rightarrow \mathbb{R}$ constant on the orbits of $N$. Assume further than $\frac{\delta H}{\delta M}(M)=H_{-1}+H_{0}+H_{1}$ is the splitting induced by (3.4) with $H_{-1}=v_{r}$. Then, the evolution induced by (3.5) on $\mathbf{k}$ is Hamiltonian with respect to the reduction of (3.1) and its associated Hamiltonian is $h$.

For more details on geometric Poisson brackets see [M1].

\section{The case $G=O(p+1, q+1)$ and Complexly coupled KdV equations}

In this section we study the case of $G=O(p+1, q+1)$ acting on $\mathbb{R}^{p+q}$ as described in [Oc]. Using the gradation (3.4) as appearing in $[\mathrm{K}]$ we can locally factor an element of the group as $g=g_{1} g_{0} g_{-1}$, with $g_{i} \in G_{i}$ where

$$
\begin{gathered}
g_{1}(Z)=\left(\begin{array}{cccc}
1-\frac{1}{2}\|Z\|^{2} & Z_{1}^{T} & \frac{1}{2}\|Z\|^{2} & Z_{2}^{T} \\
-Z_{1} & I_{p} & Z_{1} & 0 \\
-\frac{1}{2}\|Z\|^{2} & Z_{1}^{T} & 1+\frac{1}{2}\|Z\|^{2} & Z_{2}^{T} \\
Z_{2} & 0 & -Z_{2} & I_{q}
\end{array}\right), g_{0}(a, b, \Theta)=\left(\begin{array}{cccc}
a & 0 & b & 0 \\
0 & \Theta_{11} & 0 & \Theta_{12} \\
b & 0 & a & 0 \\
0 & \Theta_{21} & 0 & \Theta_{22}
\end{array}\right), \\
g_{-1}(Y)=\left(\begin{array}{cccc}
1-\frac{1}{2}\|Y\|^{2} & -Y_{1}^{T} & -\frac{1}{2}\|Y\|^{2} & Y_{2}^{T} \\
Y_{1} & I_{p} & Y_{1} & 0 \\
\frac{1}{2}\|Y\|^{2} & Y_{1}^{T} & 1+\frac{1}{2}\|Y\|^{2} & -Y_{2}^{T} \\
Y_{2} & 0 & Y_{2} & I_{q}
\end{array}\right) .
\end{gathered}
$$

The matrix $I_{r}$ is the $r \times r$ identity matrix, $Z=\left(\begin{array}{c}Z_{1} \\ Z_{2}\end{array}\right), Y=\left(\begin{array}{c}Y_{1} \\ Y_{2}\end{array}\right),\|X\|^{2}=X^{T} J X$ with $J=\left(\begin{array}{cc}I_{p} & 0 \\ 0 & -I_{q}\end{array}\right)$ and also $\Theta=\left(\begin{array}{cc}\Theta_{11} & \Theta_{12} \\ \Theta_{21} & \Theta_{22}\end{array}\right) \in O(p, q), a^{2}-b^{2}=1$. The 
corresponding splitting in the algebra is given by

$$
\begin{gathered}
V_{1}(z)=\left(\begin{array}{cccc}
0 & z_{1}^{T} & 0 & z_{2}^{T} \\
-z_{1} & 0 & z_{1} & 0 \\
0 & z_{1}^{T} & 0 & z_{2}^{T} \\
z_{2} & 0 & -z_{2} & 0
\end{array}\right), V_{0}(\alpha, A)=\left(\begin{array}{cccc}
0 & 0 & \alpha & 0 \\
0 & A_{11} & 0 & A_{12} \\
\alpha & 0 & 0 & 0 \\
0 & A_{21} & 0 & A_{22}
\end{array}\right), \\
V_{-1}(y)=\left(\begin{array}{cccc}
0 & -y_{1}^{T} & 0 & y_{2}^{T} \\
y_{1} & 0 & y_{1} & 0 \\
0 & y_{1}^{T} & 0 & -y_{2}^{T} \\
y_{2} & 0 & y_{2} & 0
\end{array}\right),
\end{gathered}
$$

where $y=\left(\begin{array}{l}y_{1} \\ y_{2}\end{array}\right), z=\left(\begin{array}{l}z_{1} \\ z_{2}\end{array}\right)$ and where $A=\left(A_{i j}\right) \in \mathfrak{o}(p, q)$. The algebra structure can be described as

$$
\begin{gathered}
{\left[V_{0}(\alpha, A), V_{1}(z)\right]=V_{1}(J A J z+\alpha z), \quad\left[V_{0}(\alpha, A), V_{-1}(y)\right]=V_{-1}(A y-\alpha y),} \\
{\left[V_{1}(z), V_{-1}(y)\right]=2 V_{0}\left(z^{T} y,\left(\begin{array}{cc}
z_{1} y_{1}^{T}-y_{1} z_{1}^{T} & -z_{1} y_{2}^{T}-y_{1} z_{2}^{T} \\
-z_{2} y_{1}^{T}-y_{2} z_{1}^{T} & z_{2} y_{2}^{T}-y_{2} z_{2}^{T}
\end{array}\right)\right)} \\
{\left[V_{0}(\alpha, A), V_{0}(\beta, B)\right]=V_{0}(0,[A, B]) .}
\end{gathered}
$$

With this factorization one chooses $H=G_{0} \cdot G_{1}$ and uses $G_{-1}$ as a local section of $G / H$. As it is the case for any homogenous space, the action of $G$ on $G / H$ is uniquely determine by the relation

$$
g g_{-1}(u)=g_{-1}(g \cdot u) h
$$

where $h \in H$. If $g=g_{1}(Z) g_{0}(a, b, \Theta) g_{-1}(Y)$, the above condition gives us the formula for the action

$$
g \cdot u=\frac{(a-b) \Theta(u+Y)+(a-b)^{2}\|u+Y\|^{2} J Z}{1+(a-b)^{2}\|u+Y\|^{2}\|Z\|^{2}+2(a-b) Z^{T} \Theta(u+Y)}
$$

for any $u \in \mathbb{R}^{p+q}$. Notice that this can be written as $g \cdot u=L(u)\left(1+Z^{T} L(u)\right)^{-1}$, where $L(u)=(a-b) \Theta(u+Y)+(a-b)^{2}\|u+Y\|^{2} J Z$.

4.1. Classification of differential invariants of curves in $O(p+1, q+1) / H$. In this subsection we use Fels and Olvers moving frame method to find a generating system of independent differential invariants for curves in $\mathbb{R}^{p+q}$ under the action of $O(p, q)$. The result is similar to the one obtained in [M2] in the conformal case (the case $q=0$ ) and some of the notation is taken from there. Using normalization equations we will find a right invariant moving frame for the action. Locally, the moving frame can be written as $\rho=\rho_{1} \rho_{0} \rho_{-1}$, with $\rho_{i} \in G_{i}$. We will then find the left invariant Serre-Frenet equation for the moving frame, $K=-\rho_{x} \rho^{-1}$. We will show that, if $K=K_{1}+K_{0}+K_{-1}$ according to the gradation (3.4), then $K_{-1}$ is constant, $K_{1}$ contains in its entries two independent differential invariants of third order, and the rest $p+q$ invariants are generated by the linear action of $O(p, q)$ on $\mathbb{R}^{p+q}$ and can be obtained using a classical moving frame. They generate the entries of $K_{0}$. We call the two generators in $K_{1}$ differential invariants of projective-type.

First of all, let's describe the first three normalization equations and its consequences. We will then describe the rest of the invariants looking at the remaining 
normalization equations. Clearly, from (4.4) the zero order normalization equation

$$
g \cdot u=0
$$

can be solved by choosing

$$
Y=-u \text {. }
$$

This determines the $\rho_{-1}$ factor of the right invariant frame, that is $\rho_{-1}=g_{-1}(-u)$. The first normalization equation is obtained by differentiating equation (4.4) with respect to $x$ and substituting $Y=-u$ after the differentiation has been completed. That is

$$
g \cdot u^{(1)}=(a-b) \Theta u_{1}=e_{1}
$$

where $e_{i}$ will be the standard unit vectors in $\mathbb{R}^{p+q}\left(c_{1}=e_{1}\right)$. Generically (for example, whenever $\left.\|u\|^{2}>0\right)$ the equation has maximal rank and determines

$$
a-b=\left\|u_{1}\right\|^{-1}, \Theta^{-1} e_{1}=\frac{u_{1}}{\left\|u_{1}\right\|} .
$$

We would need to choose $e_{p+2}$ or $e_{p+q}$ instead of $e_{1}$ if $\left\|u_{1}\right\|<0$ but the rest of the results would be completely analogous. We now proceed to the second normalization equation. Again, we need to differentiate the action (4.4) with respect to $x$ twice, and then substitute the values obtained in the previous two equations. The result equals zero $\left(c_{2}=0\right)$ will be our second normalization equation. It is given by

where $\tilde{I}=\left(\begin{array}{cc}-1 & \mathbf{0}^{T} \\ \mathbf{0} & I_{p+q-1}\end{array}\right)$. This equation determines

$$
Z=-\frac{1}{2} \tilde{I} J \Theta \frac{u_{2}}{\left\|u_{1}\right\|}
$$

and with it the $\mathfrak{g}_{1}$-factor of the moving frame $\rho_{1}=g_{1}(Z)$. Finally we look at the rest of the normalization equations and at how they will determine the remaining columns of $\Theta^{-1}$ and with it the last factor of the moving frame $\rho_{0}$. We will explicitly write out the third normalization equation and describe the others. Notice that up to this point all normalization equations have maximal rank and therefore there are no zero, first or second order nontrivial differential invariants. Repeating the process we obtain the third normalization equation by differentiating three times the action, substituting the values obtained through previous normalizations and making it equal to a vector with as many constant entries as possible. The equation thus produced does not have maximum rank though and so we cannot make it equal to a constant vector. Indeed, the vector we equate it to will determine the second column of $\Theta^{-1}$, but this column needs to be orthogonal to the first column, and also needs to be a unit vector (the inner product is always given by $J$ ). That means the rank is two less than the maximum. We can then write

$$
\Theta \frac{u_{3}}{\left\|u_{1}\right\|}+6 p_{12} J Z+e_{1}\left(6\|Z\|^{2}-24\left(Z^{T} e_{1}\right)^{2}\right)=k_{1} e_{1}+k_{2} e_{2} .
$$

where $p_{i j}=\frac{\left\langle u_{i}, u_{j}\right\rangle}{\left\|u_{1}\right\|^{2}}$ is defined similarly to the conformal case in [M2]. The difference here is that $\langle$,$\rangle and \|\|^{2}$ are determined by the $J$-inner product. Inverting $\Theta$ and substituting previous values for $\Theta^{-1} e_{1}$ we get an expression for $\Theta^{-1} e_{2}$. If we then impose on $\Theta^{-1} e_{2}$ the condition of being orthogonal to $\Theta^{-1} e_{1}$ and a unit 
vector, we determine the value of the first two differential invariants $k_{1}$ and $k_{2}$. They are given by

$$
\begin{gathered}
k_{1}=p_{13}+\frac{3}{2} p_{22}-3 p_{12}^{2} \\
k_{2}=p_{33}-6 p_{23} p_{12}-p_{13}^{2}+6 p_{13} p_{12}^{2}+9 p_{22} p_{12}^{2}-9 p_{12}^{4} .
\end{gathered}
$$

These are similar to the formulas found for the two third order conformal differential invariants in [M1]. In that case $k_{2}^{\frac{1}{4}}$ was the conformal arc-length, and its behavior under changes of the variable $x$ also makes it a good candidate for arc-length choice in our case. With these two invariants already determined, the second column of $\Theta^{-1}$ is given by

$$
\Theta^{-1} e_{2}=\frac{1}{k_{2}}\left(\frac{u_{3}}{\left\|u_{1}\right\|}-3 p_{12} \frac{u_{2}}{\left\|u_{1}\right\|}-\left(p_{13}-3 p_{12}^{2}\right) \frac{u_{1}}{\left\|u_{1}\right\|}\right)
$$

If we now look at the fourth order normalization equations we can easily observe the pattern it is created and how the remaining invariants will be formed. The fourth order equation will look like a linear combination of $\Theta \frac{u_{i}}{\left\|u_{1}\right\|}$. We can then make the equation equal to a linear combination of $e_{1}, e_{2}$ and $e_{3}$. From the recursion formula in [FO2] we know that the coefficients of $e_{1}$ and $e_{2}$ will be functions of $k_{1}, k_{2}$ and perhaps their derivatives with respect to $x$. But $\Theta^{-1} e_{3}$ will need to be orthogonal to the previous two columns and it needs to be a unit vector. That tells us that the rank of the equation above is three less than the maximum. Therefore we have one fourth order differential invariant, $\hat{k}_{3}$, the coefficient of $e_{3}$ in the fourth order normalization equation. Recursion formulas in [FO2] imply that this equation will be equal to $\left(k_{1}\right)_{x} e_{1}+\left(k_{2}\right)_{x} e_{2}+\hat{k}_{3} e_{3}$. We proceed this way until we determine $\Theta^{-1} e_{p+q}$, which will define one last new differential invariant $\hat{k}_{p+q}$. The fact that we use $J$ instead of $I$ simply introduces a negative in the definition of some invariants. Since we work in generic cases, this represents no impediment to our construction. Notice that we need $\hat{k}_{r} \neq 0$, for all $r$ to define $\Theta^{-1}$. We will be back to this point in the next subsection. Now we have proved the following Theorem.

Theorem 8. A complete set of generating and independent differential invariants for curves in $O(p+1, q+1) / H$ is given by $k_{1}, k_{2}$ and $\hat{k}_{i}, i=3, \ldots, p+q$ defined above. The first two invariants have order 3 and $\hat{k}_{i}$ has order $i+1$ for $i=3, \ldots, p+q$.

The last part of this subsection is the description of the Serre-Frenet equations for a moving frame conveniently obtained from the one above. To be able to carry out the study of the associated Poisson brackets, we will need to change the $\rho_{0}$ factor of the moving frame we found. We will show that the third order differential invariants appear in the $\mathfrak{g}_{1}$ component of the equation, while the rest appears in the $\mathfrak{g}_{0}$ component. First a lemma to determine a different choice of $\Theta^{-1}$. Recall that in [M1] it was proved that the $G_{0}$ component of any left invariant moving frame, as an element of $\mathrm{GL}(p+q, \mathbb{R})$ acting on $\mathfrak{g}_{-1}$ under the adjoint action, has in columns an independent set of invariant vectors along the curve. That is, what it is known as a classical moving frame. 
Lemma 1. We can choose a classical moving frame $\frac{F_{i}}{\left\|u_{1}\right\|}, i=1, \ldots, p+q$ such that $\left\langle\frac{F_{i}}{\left\|u_{1}\right\|}, \frac{F_{j}}{\left\|u_{1}\right\|}\right\rangle=\delta_{i}^{j}$ and such that, if $\Omega=\left(\frac{F_{2}}{\left\|u_{1}\right\|}, \ldots, \frac{F_{p+q}}{\left\|u_{1}\right\|}\right)$ then

$$
\Omega^{T} J \Omega_{x}=\left(\begin{array}{ccccccc}
0 & -\kappa_{3} & \ldots & -\kappa_{p} & -\kappa_{p+1} & \ldots & -\kappa_{p+q} \\
\kappa_{3} & 0 & 0 & 0 & \ldots & 0 & 0 \\
\vdots & \vdots & \vdots & \vdots & \vdots & \vdots & \vdots \\
\kappa_{p} & 0 & 0 & 0 & \ldots & 0 & 0 \\
-\kappa_{p+1} & 0 & 0 & 0 & \ldots & 0 & 0 \\
-\kappa_{p+2} & 0 & 0 & 0 & \ldots & 0 & 0 \\
\vdots & \vdots & \vdots & \vdots & \vdots & \vdots & \vdots \\
-\kappa_{p+q} & 0 & 0 & 0 & \ldots & 0 & 0
\end{array}\right)
$$

with $k_{1}, k_{2}, \kappa_{i}, i=3, \ldots, p+q$ forming a complete set of generating differential invariants for the curve.

In fact, the procedure we will describe below is merely a choice to obtain a matrix looking like the one in the statement of the lemma. One can choose several different procedures and obtain different matrices. This was thoroughly explained in [M2]. In this lemma we choose the best suited for the calculations that follow.

Proof. Similarly to what was done for the Möbius sphere, choose $F_{1}=\left\|u_{1}\right\| \Theta^{-1} e_{1}$ and $F_{2}=\left\|u_{1}\right\| \Theta^{-1} e_{2}$ as determined by the normalization equations. We find $\left(F_{2}\right)_{x}$, an invariant vector, and we project it on $F_{1}^{\perp}$. We call the vector $\widehat{\left(F_{2}\right)_{x}}$. We choose

$$
\kappa_{3}^{2}=\left\langle\widehat{\left(F_{2}\right)_{x}}, \widehat{\left(F_{2}\right)_{x}}\right\rangle
$$

and we define $\tilde{F}_{3}=\frac{1}{\kappa_{3}} \widehat{\left(F_{2}\right)_{x}}$. It is immediate that $\kappa_{3}$ is an invariant and $\tilde{F}_{3}$ is an invariant vector. We now consider $\widehat{\left(\tilde{F}_{3}\right)_{x}}+\kappa_{3} F_{2}, J$-orthogonal to $F_{1}, F_{2}$ and $\tilde{F}_{3}$. We call

$$
\kappa_{4}^{2}=\left\langle\widehat{\left(F_{3}\right)_{x}}+\kappa_{3} F_{2}, \widehat{\left(F_{3}\right)_{x}}+\kappa_{3} F_{2}\right\rangle
$$

and define $\tilde{F}_{4}=\frac{1}{\kappa_{4}} \widehat{\left(F_{3}\right)_{x}}+\kappa_{3} F_{2}$. And so on. Clearly, this procedure generates a classical moving frame along $u$. Namely $\left\{F_{1}, F_{2}, \tilde{F}_{3}, \ldots, \tilde{F}_{p+q}\right\}$. But the matrix $\tilde{\Omega}=\frac{1}{\left\|u_{1}\right\|}\left(F_{2}, \tilde{F}_{3}, \ldots, \tilde{F}_{p+q}\right)$ would not be the one in the lemma, rather it will look like the classical Serret-Frenet equations in Riemannian geometry. Furthermore, if we were to use this frame instead of the frame shown in the lemma, the geometric Poisson brackets would be described in such a way that we would not be able to find a clear restriction to the space of differential invariants of projective type, as we will see later. Hence, we now describe the so-called natural frame. For this, we choose

$$
\left(F_{1}, F_{2}, \ldots, F_{p+q}\right)=\left(F_{1}, F_{2}, \tilde{F}_{3}, \ldots, \tilde{F}_{p+q}\right)\left(\begin{array}{ll}
1 & 0 \\
0 & \theta
\end{array}\right) .
$$

As it was explained in [M2], $\theta(x) \in O(p-2, q)$ can be solved to obtain a frame with the properties in the statement of the lemma. The resulting set of differential invariants, $\kappa_{r}, r=3, \ldots, p+q$ are usually called natural curvatures. We refer the reader to [M2] for the complete description and explanations. Notice that, although the natural moving frame generates a complete set of invariants, these are non-local in nature. Still, one can use geometric brackets effectively to obtain what we look for. Also, notice that we are assuming $p>1$. If $p \leq 1$ one only needs minimal changes to the proof above. 
This lemma determines a classical frame and, from Theorem 5 , the $G_{0}$ component of a moving frame with $a-b=\left\|u_{1}\right\|$ (this was determined by the first normalization equation) and $\Theta^{-1}=\left(F_{1}, F_{2}, \ldots, F_{p+q}\right)$. In fact, this frame can be merely produced by choosing appropriate normalization equations of order fourth and higher. From now on we choose $\rho=\rho_{1} \rho_{0} \rho_{-1}$, with $\rho_{1}$ and $\rho_{-1}$ determined by the first three normalization equations and $\rho_{0}$ as determined by the lemma.

Theorem 9. The Serre-Frenet equation for $\rho$ determined as above is given by $-\rho_{x} \rho^{-1}=K=K_{1}+K_{0}+K_{-1}$ where $K_{i} \in \mathfrak{g}_{i}$ are given by

$$
\begin{aligned}
& V_{-1}\left(e_{1}\right)=K_{-1}=\left(\begin{array}{cccc}
0 & -e_{1}^{T} & 0 & 0 \\
e_{1} & 0 & e_{1} & 0 \\
0 & e_{1}^{T} & 0 & 0 \\
0 & 0 & 0 & 0
\end{array}\right) \\
& V_{0}\left(\alpha_{0}, \hat{K}_{0}\right)=K_{0}=\left(\begin{array}{cccc}
0 & 0 & \alpha_{0} & 0 \\
0 & A_{0} & 0 & B_{0} \\
\alpha_{0} & 0 & 0 & 0 \\
0 & B_{0}^{T} & 0 & 0
\end{array}\right) \\
& V_{1}\left(\kappa_{1} e_{1}+\kappa_{2} e_{2}\right)=K_{1}=\left(\begin{array}{cccc}
0 & \kappa_{1} e_{1}^{T}+\kappa_{2} e_{2}^{T} & 0 & 0 \\
-\left(\kappa_{1} e_{1}+\kappa_{2} e_{2}\right) & 0 & \kappa_{1} e_{1}+\kappa_{2} e_{2} & 0 \\
0 & \kappa_{1} e_{1}^{T}+\kappa_{2} e_{2}^{T} & 0 & 0 \\
0 & 0 & 0 & 0
\end{array}\right),
\end{aligned}
$$

where $\alpha_{0}=-p_{12}$ and where

$$
A_{0}=\left(\begin{array}{cccccc}
0 & 0 & 0 & 0 & \ldots & 0 \\
0 & 0 & -\kappa_{3} & -\kappa_{4} & \ldots & -\kappa_{p} \\
0 & \kappa_{3} & 0 & 0 & \ldots & 0 \\
\vdots & \vdots & \vdots & \vdots & \vdots & 0 \\
0 & \kappa_{p} & 0 & \ldots & 0 & 0
\end{array}\right), B_{0}=\left(\begin{array}{cccc}
0 & 0 & \ldots & 0 \\
-\kappa_{p+1} & -\kappa_{p+2} & \ldots & -\kappa_{p+q} \\
0 & 0 & \ldots & 0 \\
\vdots & \vdots & \vdots & \vdots \\
0 & 0 & \ldots & 0
\end{array}\right)
$$

Proof. The proof of this theorem is a simple calculation. The right invariant SerreFrenet equations are given by $\rho_{x} \rho^{-1}$, which, in terms of the factorization we have used become

$$
\rho_{1} \rho_{0}\left(\left(\rho_{-1}\right)_{x} \rho_{-1}^{-1}\right) \rho_{0}^{-1} \rho_{1}^{-1}+\rho_{1}\left(\left(\rho_{0}\right)_{x} \rho_{0}^{-1}\right) \rho_{1}^{-1}+\left(\rho_{1}\right)_{x} \rho_{1}^{-1} .
$$

Using the values we found in the previous theorem and after some simplifications we get that

$$
\begin{gathered}
\rho_{1} \rho_{0}\left(\left(\rho_{-1}\right)_{x} \rho_{-1}^{-1}\right) \rho_{0}^{-1} \rho_{1}^{-1}=V_{-1}\left(-e_{1}\right) \\
+V_{0}\left(-2 Z_{1}^{T} e_{1},\left(\begin{array}{cc}
-2 Z_{1} e_{1}^{T}+2 e_{1} Z_{1}^{T} & 2 e_{1} Z_{2}^{T} \\
2 Z_{2} e_{1}^{T} & 0
\end{array}\right)\right)+V_{1}\left(2 Z e_{1}^{T} Z-\|Z\|^{2} e_{1}\right),
\end{gathered}
$$

where $V_{i}$ is given as in (4.2). Similarly, the term $\rho_{1}\left(\left(\rho_{0}\right)_{x} \rho_{0}^{-1}\right) \rho_{1}^{-1}$ is given by

$$
\left.\rho_{1}\left(\left(\rho_{0}\right)_{x} \rho_{0}^{-1}\right) \rho_{1}^{-1}=V_{1}\left(\left(\Theta_{x} \Theta^{-1}\right)^{T} Z-\left(a b_{x}-b a_{x}\right) Z\right)+V_{0}\left(a b_{x}-b a_{x}, \Theta_{x} \Theta^{-1}\right)\right) .
$$

It is trivial to check that $\left(\rho_{1}\right)_{x} \rho_{1}^{-1}$ is given by the $\mathfrak{g}_{1}$ component $V_{1}\left(Z_{x}\right)$. 
G. MARÍ BEFFA

Putting these three terms together we get

$$
\begin{gathered}
\rho_{x} \rho^{-1}=V_{-1}\left(-e_{1}\right)+V_{1}\left(2 Z e_{1}^{T} Z-\|Z\|^{2} e_{1}+\left(\Theta_{x} \Theta^{-1}\right)^{T} Z-\left(a b_{x}-b a_{x}\right) Z+Z_{x}\right) \\
+V_{0}\left(-2 Z_{1}^{T} e_{1}+a b_{x}-b a_{x}, \Theta_{x} \Theta^{-1}+\left(\begin{array}{cc}
-2 Z_{1} e_{1}^{T}+2 e_{1} Z_{1}^{T} & 2 e_{1} Z_{2}^{T} \\
2 Z_{2} e_{1}^{T} & 0
\end{array}\right)\right) .
\end{gathered}
$$

Clearly, $K_{-1}=-V_{-1}\left(-e_{1}\right)$. Using $a-b=\left\|u_{1}\right\|^{-1}$ and $a^{2}-b^{2}=1$ we can directly find that $a b_{x}-b a_{x}=p_{12}$. Also, using the second normalization equation (and assuming $p>0$, some minor changes need to be introduced if $p=0$ ) we get that $Z^{T} e_{1}=Z_{1}^{T} e_{1}=\frac{1}{2} p_{12}$ and $\|Z\|^{2}=\frac{1}{4} p_{22}$. (There is some abuse of notation here. We are using $e_{1}$ for both $e_{1} \in \mathbb{R}^{p}$ and $e_{1} \in \mathbb{R}^{p+q}$, but it will always be determined by the context.)

Recall from the Lemma that

$$
\Theta^{-1}=\frac{1}{\left\|u_{1}\right\|}\left(\begin{array}{llll}
F_{1} & F_{2} & \ldots & F_{p+q}
\end{array}\right)=\left(\begin{array}{cc}
\frac{F_{1}}{\left\|u_{1}\right\|} & \Omega
\end{array}\right)=J \Theta^{T} J \in O(p, q) .
$$

Therefore

$$
\Theta_{x} \Theta^{-1}=J\left(\begin{array}{c}
\left(\frac{F_{1}^{T}}{\left\|u_{1}\right\|}\right)_{x} \\
\Omega_{x}^{T}
\end{array}\right) J\left(\begin{array}{cc}
\frac{F_{1}}{\left\|u_{1}\right\|} & \Omega
\end{array}\right)=J\left(\begin{array}{cc}
\left(\begin{array}{c}
F_{1}^{T} \\
\left\|u_{1}\right\|
\end{array}\right)_{x} J \frac{F_{1}}{\left\|u_{1}\right\|} & \left(\begin{array}{c}
F_{1}^{T} \\
\left\|u_{1}\right\|
\end{array}\right)_{x} J \Omega \\
\Omega_{x}^{T} \frac{F_{1}}{\left\|u_{1}\right\|} & \Omega_{x}^{T} J \Omega
\end{array}\right) .
$$

The vector $\frac{F_{1}}{\left\|u_{1}\right\|}$ is a $J$-unit vector and so $\left(\frac{F_{1}^{T}}{\left\|u_{1}\right\|}\right)_{x} J \frac{F_{1}}{\left\|u_{1}\right\|}=0$. Also, notice that from the second normalization equations

$$
Z=\left(\begin{array}{c}
Z_{1} \\
Z_{2}
\end{array}\right)=-\frac{1}{2} \tilde{I} J \Theta \frac{u_{2}}{\left\|u_{1}\right\|}=\left(\begin{array}{c}
\frac{1}{2} \frac{F_{1}^{T}}{\left\|u_{1}\right\|} \\
-\frac{1}{2} \Omega^{T}
\end{array}\right) J \frac{u_{2}}{\left\|u_{1}\right\|}=\left(\begin{array}{c}
\frac{1}{2}\left\langle\frac{F_{1}^{T}}{\left\|u_{1}\right\|}, \frac{u_{2}}{\left\|u_{1}\right\|}\right\rangle \\
-\frac{1}{2} \Omega^{T} J \frac{u_{2}}{\left\|u_{1}\right\|}
\end{array}\right) .
$$

Therefore, $2 Z_{2}^{T}=-\frac{u_{2}^{T}}{\left\|u_{1}\right\|} J \Omega$. Given that $\frac{F_{1}^{T}}{\left\|u_{1}\right\|} J \Omega=0$ we can conclude that $\left(\frac{F_{1}^{T}}{\left\|u_{1}\right\|}\right)_{x} J \Omega+$ $2 Z_{2}^{T}=\frac{u_{2}^{T}}{\left\|u_{1}\right\|} J \Omega-\frac{u_{2}^{T}}{\left\|u_{1}\right\|} J \Omega=0$.

The component $K_{0} \in \mathfrak{g}_{0}$ is thus given by the negative of

$$
\begin{gathered}
V_{0}\left(a b_{x}-b a_{x}-2 Z_{1}^{T} e_{1}, \Theta_{x} \Theta^{-1}+\left(\begin{array}{cc}
-2 Z_{1} e_{1}^{T}+2 e_{1} Z_{1}^{T} & 2 Z_{2}^{T} \\
2 Z_{2} & 0
\end{array}\right)\right) \\
=V_{0}\left(0, J\left(\begin{array}{cc}
0 & 0 \\
0 & \Omega^{T} J \Omega_{x}
\end{array}\right)\right)
\end{gathered}
$$

where $\Omega$ is given as in the lemma. Direct application of the Lemma results in the value of $K_{0}$ in the statement of the Theorem with $\kappa_{i}=\hat{k}_{i}, i=3, \ldots, p+q$.

Finally, the $\mathfrak{g}_{1}$ component is defined by $Z_{x}+2 Z e_{1}^{T} Z-\|Z\|^{2} e_{1}+\left(\Theta_{x} \Theta^{-1}\right)^{T} Z-$ $\left(a b_{x}-b a_{x}\right) Z$. Differentiating the second normalization equation and using the third one we obtain the following formula

$$
Z_{x}=-\hat{I}\left(\Theta_{x} \Theta^{-1}\right)^{T} \hat{I} Z+2 p_{12} Z-\frac{3}{4} p_{22} e_{1}-\frac{1}{2} \hat{I} J\left(k_{1} e_{1}+k_{2} e_{2}\right) .
$$

From above we can conclude that $\left(\Theta_{x} \Theta^{-1}\right)^{T} Z-\hat{I}\left(\Theta_{x} \Theta^{-1}\right)^{T} \hat{I} Z=-2 p_{12} Z+p_{22} e_{1}$. If we now put everything together we get that

$$
K_{1}=V_{1}\left(-\frac{1}{2} \hat{I} J\left(k_{1} e_{1}+k_{2} e_{2}\right)\right)
$$


Choosing $\kappa_{1}=-\frac{1}{2} k_{1}$ and $\kappa_{2}=\frac{1}{2} k_{2}$ the proof is now completed. Notice that we are assuming $p>1$ but in other cases one only needs a couple of minor modifications in the signs.

4.2. Complexly coupled $\mathbf{K d V}$ equations associated to $O(p+1, q+1) / H$. In this subsection we will show that the Poisson geometric brackets associated to $O(p+1, q+1) / H$ and defined on matrices of the form (4.8) can be reduced to the submanifold $K_{0}=0$ to obtain two Hamiltonian structures for the wellknown complexly coupled $\mathrm{KdV}$ equations. We will then give explicitly the geometric evolution of curves on $O(p+1, q+1) / H$ that, when proper initial conditions are chosen, induces a system of complexly coupled $\mathrm{KdV}$ equations on $\kappa_{1}$ and $\kappa_{2}$, the invariants of projective type. These evolutions will have to be understood as a limit evolution. Indeed the submanifold $K_{0}=0$ can be considered algebraically and a Poisson restriction can be performed. But if we think geometrically, such submanifold does not make sense since we are assuming the invariants appearing in $K_{0}$ not to vanish. Nevertheless, we will see how geometric evolutions that depend only on $F_{1}$ and $F_{2}$ (the members of the frame that were generated without the use of $\hat{k}_{i}, i=3, \ldots, p+q$ ) have a well defined limit as $K_{0} \rightarrow 0$. That is, a complexly coupled system of $\mathrm{KdV}$ equations is a level set of the invariant evolution. Our evolution will depend only on $F_{1}$ and $F_{2}$. Our next proposition follows from a well-known classical result, we are simply using the classical moving frame we just found.

Proposition 1. Let

$$
u_{t}=F\left(u, u_{1}, u_{2}, \ldots,\right)
$$

be a curve evolution with $u: U \subset \mathbb{R}^{2} \rightarrow O(p+1, q+1) / H$ invariant under the action of $O(p+1, q+1)$, that is, the group takes solutions to solutions. Then,

$$
F=(a+b) \Theta^{-1} \mathbf{r}=\sum_{i=1}^{p+q} r_{i} F_{i}
$$

where $\Theta, a+b$ and $F_{i}$ are as in the previous subsection and where $\mathbf{r}=\left(r_{i}(\kappa)\right) \in \mathbb{R}^{p+q}$ is a differential invariant vector, that is a vector whose entries are functions of $\kappa_{i}$, $i=1,2, \ldots, p+q$ and their derivatives with respect to $x$.

Theorem 10. Consider the geometric Poisson brackets associated to $O(p+1, q+$ 1) $/ H$ defined on matrices of the form (4.8). Then, the brackets are well defined for matrices with $K_{0}=0$. Furthermore, the brackets reduce to the submanifold $K_{0}=0$ to produce two compatible Hamiltonian structures usually associated to the integration of a system of complexly coupled KdV equations.

Proof.

In order to prove this theorem we will write the geometric bracket in a way that we can use.

Let $\mathfrak{n}$ be given as in the construction of the geometric bracket. That is, $\mathfrak{n}=\mathfrak{g}_{1} \oplus \mathfrak{n}_{0}$ with $n_{0}=\operatorname{Stab}\left(K_{-1}\right) \subset \mathfrak{g}_{0}$. Let $f, h: \mathcal{K} \rightarrow \mathbb{R}$ be two functionals on the space of differential invariants and let $\mathcal{F}, \mathcal{H}$ be two extensions such that if $F=\frac{\delta \mathcal{F}}{\delta L}$ and $H=\frac{\delta \mathcal{H}}{\delta L}$, then

$$
H^{\prime}+[K, H], F^{\prime}+[K, F] \in \mathfrak{n}^{0}
$$


where $\mathfrak{n}^{0}$ is the annihilator of $\mathfrak{n}$. This is the condition imposed by $\mathcal{F}$ and $\mathcal{H}$ being constant on the $N$-orbits. It is not hard to check that

$$
\mathfrak{n}_{0}=\left\{V_{0}(0, A), \text { such that } A e_{1}=0\right\}
$$

and hence

$$
\mathfrak{n}_{0}^{0}=\left\{V_{0}(\alpha, B), \text { such that } B e_{k}=0, k \neq 1\right\} .
$$

Let $H=H_{-1}+H_{0}+H_{1}$ be given by the gradation. Assume $H_{-1}=V_{-1}(\mathbf{h})$, $\mathbf{h}=\left(h_{1}, h_{2}, \ldots, h_{p+q}\right)^{T}$, and $H_{0}=V_{0}\left(\alpha^{h}, A^{h}\right)$. From (4.9) we obtain

$$
\begin{aligned}
H_{-1}^{\prime}+\left[K_{-1}, H_{0}\right]+\left[K_{0}, H_{-1}\right] & =0 \\
H_{0}^{\prime}+\left[K_{0}, H_{0}\right]+\left[K_{1}, H_{-1}\right]+\left[K_{-1}, H_{1}\right] & \in \mathfrak{n}_{0}^{0} .
\end{aligned}
$$

The geometric Poisson bracket is then given by

$$
\begin{aligned}
\{f, h\}(K) & =\int_{S^{1}}\left\langle H_{0}^{\prime}+\left[K_{0}, H_{0}\right]+\left[K_{1}, H_{-1}\right]+\left[K_{-1}, H_{1}\right], F_{0}\right\rangle d x \\
& +\int_{S^{1}}\left\langle H_{1}^{\prime}+\left[K_{1}, H_{0}\right]+\left[K_{0}, H_{1}\right], F_{-1}\right\rangle d x .
\end{aligned}
$$

Assume that $F_{0}=\widehat{F_{0}}+F_{0}^{0}$, where $\widehat{F_{0}} \in \mathfrak{n}_{0}$ and $F_{0}^{0} \in \mathfrak{n}_{0}^{0}$ (or, in general, $F_{0}^{0}$ belongs to a complement of $\mathfrak{n}_{0}$ in $\mathfrak{g}_{0}$ as vector subspaces). Then, from equations (4.10), the geometric bracket depends only on $H_{0}^{0}$ and $F_{0}^{0}$. Also, since $F_{-1}^{\prime}+\left[K_{-1}, F_{0}\right]+$ $\left[K_{0}, F_{-1}\right]=0$ we have that the bracket can be written as

$$
\begin{aligned}
\{f, h\}(K) & =\int_{S^{1}}\left\langle\left(H_{0}^{0}\right)^{\prime}+\left[K_{0}, H_{0}^{0}\right], F_{0}^{0}\right\rangle d x \\
& +\int_{S^{1}}\left(\left\langle\left[K_{1}, H_{-1}\right], F_{0}^{0}\right\rangle-\left\langle H_{0}^{0},\left[K_{1}, F_{-1}\right]\right\rangle\right) d x
\end{aligned}
$$

where $H_{0}^{0}, F_{0}^{0}, H_{-1}$ and $F_{-1}$ are determined completely by the equations in (4.10). In fact the first equation determines $H_{0}^{0}$ in terms of $K_{-1}$ and $\left[K_{0}, H_{-1}\right]$ and the second equation determines the component of $\left[K_{1}, H_{-1}\right]$ needed in the bracket in terms of $\left[K_{-1}, H_{1}\right], H_{0}^{0}$ and $\left[K_{0}, H_{0}\right]$.

Finally, we clearly see that one can take the limit as $K_{0} \rightarrow 0$. Then, from the first equation in (4.10) we can solve for $H_{0}^{0}$ completely in terms of $H_{-1}$ and the result is well defined in the limit . Also, since $\left[K_{-1}, H_{1}\right] \in \mathfrak{n}_{0}^{0}$ always, the second equation in (4.10) becomes $H_{0}^{\prime}+\left[K_{1}, H_{-1}\right] \in \mathfrak{n}_{0}^{0}$ in the limit which further relates $H_{-1}$ and $H_{0}$.

Let $\mathcal{K}_{1} \subset \mathcal{K}$ be the algebraic subset for which $K_{0}=0$.

After this rewriting we will show that, if $h$ is a functional on $\mathcal{K}$ constant on $K_{0}$, and $f$ is a functional on $\mathcal{K}$ constant on $K_{1}$, then $\left.\{f, h\}\right|_{\mathcal{K}_{1}}=0$. This will guarantee the existence of a restriction of the limit bracket to $\mathcal{K}_{1}$. Finally we will calculate the restricted bracket to finish the proof of the Theorem.

Assume $h$ is constant on $K_{0}$ and let $\mathcal{H}$ be an extension constant on the $N$-leaves, the type we use to calculate the geometric bracket. Since $h$ and $\mathcal{H}$ coincide on $K_{0}$, the variational derivative in that direction is zero and the entries of $\widehat{H_{0}}$ in the second row and column ought to vanish. It is straightforward to check that 
$\left[K_{1}, H_{-1}\right]=2 V_{0}\left(h_{1} \kappa_{1}+h_{2} \kappa_{2}, B\right)$, where

$$
B=\left(\begin{array}{cc}
\left(\kappa_{1} e_{1}+\kappa_{2} e_{2}\right)\left(\begin{array}{c}
h_{1} \\
\vdots \\
h_{p}
\end{array}\right)^{T}-\left(\begin{array}{c}
h_{1} \\
\vdots \\
h_{p}
\end{array}\right)\left(\kappa_{1} e_{1}^{T}+\kappa_{2} e_{2}^{T}\right) & -\left(\kappa_{1} e_{1}+\kappa_{2} e_{2}\right)\left(\begin{array}{c}
h_{p+1} \\
\vdots \\
h_{p+q}
\end{array}\right)^{T} \\
-\left(\begin{array}{c}
h_{p+1} \\
\vdots \\
h_{p+q}
\end{array}\right)\left(\kappa_{1} e_{1}^{T}+\kappa_{2} e_{2}^{T}\right) & 0
\end{array}\right) .
$$

Therefore, equations (4.10) imply that, if $h$ is constant on $K_{0}$, then $H_{-1}=V_{-1}\left(h_{1} e_{1}+\right.$ $h_{2} e_{2}$ ). From the first equation in (4.10) we also get that $\alpha^{h}=-h_{1}^{\prime}$ and the first row of $A^{h}$, the matrix defining $H_{0}$, is given by $h_{2}^{\prime} e_{2}$.

Assume $f$ is constant on $K_{1}$. Then, if $F_{-1}=V_{-1}(\mathbf{f})$, the first two entries in $\mathbf{f}$ must be zero, and hence $\left\langle H_{0}^{0},\left[K_{1}, F_{-1}\right]\right\rangle=0$. Also, from the first equation in (4.10) we have that $\alpha^{f} e_{1}-A^{f} e_{1}=-\mathbf{f}^{\prime}$ and hence $\alpha^{f}$ and the first two entries of $A^{f} e_{1}$ vanish. This implies that $\left\langle\left(H_{0}^{0}\right)^{\prime}, F_{0}\right\rangle=0$. Finally, since $\left[K_{1}, H_{-1}\right]$ vanish outside the first and second rows and columns, we have $\left\langle\left[K_{1}, H_{-1}\right], F_{0}^{0}\right\rangle=0$. Putting all together we get $\left.\{f, h\}\right|_{\mathcal{K}_{1}}=0$.

Let us now calculate the restricted bracket. Assume that both $f$ and $h$ are constant on $K_{0}$. This implies

$$
\begin{gathered}
H_{-1}=V_{-1}\left(h_{1} e_{1}+h_{2} e_{2}\right), \quad F_{-1}=V_{-1}\left(f_{1} e_{1}+f_{2} e_{2}\right) \\
\alpha^{h}=-\left(h_{1}\right)_{x}, \quad \alpha^{f}=-\left(f_{1}\right)_{x}, \quad A^{h} e_{1}=\left(h_{2}\right)_{x} e_{2}, \quad A^{f} e_{1}=\left(f_{2}\right)_{x} e_{2} .
\end{gathered}
$$

Then the bracket becomes

$$
\begin{gathered}
\{f, h\}\left(K_{1}\right)=\int_{S^{1}}\left\langle V_{0}\left(\left(\alpha^{h}\right)_{x},\left(A^{h}\right)_{x}\right), V_{0}\left(\alpha^{f}, A^{f}\right)\right\rangle d x \\
+\int_{S^{1}}\left\langle\left[K_{1}, H_{-1}\right], V_{0}\left(\alpha^{f}, A^{f}\right)\right\rangle d x-\int_{S^{1}}\left\langle V_{0}\left(\alpha^{h}, A^{h}\right),\left[K_{1}, F_{-1}\right]\right\rangle d x,
\end{gathered}
$$

where $\langle$,$\rangle is half the trace of the product. Substituting the values obtained above$ for the extensions of functionals that are constant on $K_{0}$, and after trivial simplifications we get

$$
\begin{gathered}
\{f, h\}\left(\kappa_{1}, \kappa_{2}\right)=\int_{S^{1}}\left(\left(h_{1}\right)_{x x}\left(f_{1}\right)_{x}-\left(h_{2}\right)_{x x}\left(f_{2}\right)_{x}+2\left(f_{2}\right)_{x}\left(\kappa_{1} h_{2}-h_{1} \kappa_{2}\right)\right) d x \\
-\int_{S^{1}}\left(2\left(f_{1}\right)_{x}\left(h_{1} \kappa_{1}+h_{2} \kappa_{2}\right)+2\left(h_{1}\right)_{x}\left(f_{1} \kappa_{1}+f_{2} \kappa_{2}\right)-2\left(h_{2}\right)_{x}\left(\kappa_{1} f_{2}-f_{1} \kappa_{2}\right)\right) d x
\end{gathered}
$$

that is

$$
\{f, h\}\left(\kappa_{1}, \kappa_{2}\right)=-2\left(\frac{\delta h}{\delta K_{1}}\right)^{T}\left(\begin{array}{cc}
-\frac{1}{2} D^{3}+\kappa_{1} D+D \kappa_{1} & \kappa_{2} D+D \kappa_{2} \\
\kappa_{2} D+D \kappa_{2} & \frac{1}{2} D^{3}-D \kappa_{1}-\kappa_{1} D
\end{array}\right) \frac{\delta f}{\delta K_{1}} .
$$

This is equivalent to the standard Hamiltonian structure for the complexly coupled KdV system.

Let us now consider the bracket (3.3) with $\Lambda=V_{1}\left(e_{1}\right) \in \mathfrak{g}_{1}$. The reduction to $\mathcal{K}$ is then given by

$$
\{f, h\}_{0}(K)=\int_{S^{1}}\left(\left\langle\left[\Lambda, H_{0}\right], F_{-1}\right\rangle+\left\langle\left[\Lambda, H_{-1}\right], F_{0}\right\rangle\right) d x
$$


Following exactly the same procedure as before, we obtain that, if $f$ is constant on $K_{1}$ and $h$ is constant on $K_{0}$, then

$$
\left.\{f, h\}_{0}\right|_{\mathcal{K}_{1}}=0
$$

and if both $f$ and $h$ are constant on $K_{0}$, then

$$
\left.\{f, h\}_{0}\right|_{\mathcal{K}_{1}}=4\left(\frac{\delta h}{\delta K_{1}}\right)^{T}\left(\begin{array}{cc}
-D & 0 \\
0 & D
\end{array}\right) \frac{\delta f}{\delta K_{1}} .
$$

This is the invertible Poisson structure usually used to generate the recursion operator that integrates the complexly couple KdV system. This finishes the proof of the Theorem.

Theorem 11. Assume that

$$
u_{t}=r_{1} F_{1}+r_{2} F_{2}
$$

where $r_{1}$ and $r_{2}$ are differential invariants of the flow. Then, the flow induced on the differential invariants has a limit as $K_{0} \rightarrow 0$. Furthermore the limit is given by

$$
\begin{aligned}
& \left(\kappa_{1}\right)_{t}=\left(-\frac{1}{2} D^{3}+\kappa_{1} D+D \kappa_{1}\right) r_{1}+\left(\kappa_{2} D+D \kappa_{2}\right) r_{2} \\
& \left(\kappa_{2}\right)_{t}=\left(\kappa_{2} D+D \kappa_{2}\right) r_{1}+\left(\frac{1}{2} D^{3}-\kappa_{1} D-D \kappa_{1}\right) r_{2}
\end{aligned}
$$

If we choose $r_{1}=\kappa_{1}$ and $r_{2}=\kappa_{2}$, we obtain a complexly coupled system of $K d V$ equations.

Proof. Part of the proof of this theorem is essentially the same as the corresponding Theorem in [M2], so we will simply outline it.

In [M2] it was shown that, if $u$ is a solution of (4.13), then $K$ is a solution of the equation

$$
K_{t}=N_{x}+[K, N]
$$

where $N=\rho^{-1} \rho_{t}$ and where $N_{-1}=V_{-1}(\mathbf{r})$ in the gradation $N=N_{1}+N_{0}+N_{-1}$ with $\mathbf{r}=\left(r_{1}, r_{2}, 0, \ldots, 0\right)$. The above equation determines the rest of the entries in $N$ in terms of $K$ and $\mathbf{r}$ similarly to the way it was determined in [M2]. That is, if $K_{0}=V_{0}\left(0, K_{2}\right)$ with

$$
K_{2}=\left(\begin{array}{ccc}
0 & 0 & 0 \\
0 & 0 & -\kappa^{T} \\
0 & J \kappa & 0
\end{array}\right), \quad \kappa=\left(\begin{array}{c}
\kappa_{3} \\
\vdots \\
\kappa_{p+q}
\end{array}\right)
$$

the matrix $N_{0}=V_{0}\left(\alpha_{0}, A_{0}\right)$ is given by

$$
A_{0}=\left(\begin{array}{ccc}
0 & e_{1}^{T} a_{1} & \pi\left(a_{1}\right) \\
-e_{1}^{T} a_{1} & 0 & a_{2}^{T} \\
-J \pi\left(a_{1}\right) & -J a_{2} & A_{3}
\end{array}\right)
$$


and $N_{1}=V_{1}(z), K_{1}=V_{1}\left(\kappa_{1} e_{1}+\kappa_{2} e_{2}\right), K_{-1}=V_{-1}\left(e_{1}\right)$, the algebra commutation relations and straightforward calculations of the equation above gives us the values

$$
\begin{gathered}
\alpha_{0}=-\left(r_{1}\right)_{x}, \quad a_{1}=\left(\begin{array}{c}
a_{1}^{1} \\
\pi\left(a_{1}\right)
\end{array}\right)=-\left(\begin{array}{c}
\left(r_{2}\right)_{x} \\
r_{2} \kappa
\end{array}\right) \\
z^{T} e_{1}=-\frac{1}{2}\left(r_{1}\right)_{x x}+\kappa_{1} r_{1}+\kappa_{2} r_{2}, \\
\pi(z)=\frac{1}{2}\left(\begin{array}{c}
\left(r_{2}\right)_{x x} \\
\left(r_{2} \kappa\right)_{x}
\end{array}\right)-\frac{1}{2}\left(\begin{array}{c}
r_{2}\|\kappa\|^{2} \\
\left(r_{2}\right)_{x} \kappa
\end{array}\right)-\left(\begin{array}{c}
\kappa_{1} r_{2}-r_{1} \kappa_{2} \\
0
\end{array}\right) \\
a_{2}=\frac{1}{\kappa_{2}}\left(\kappa_{1} r_{2} \kappa+\left(\frac{1}{2}\left(r_{2}\right)_{x x}+\kappa_{2} r_{1}-\kappa_{1} r_{2}-\frac{1}{2} r_{2}\|\kappa\|^{2}\right) \kappa+\frac{1}{2}\left(\left(r_{2}\right)_{x} \kappa\right)_{x}-\frac{1}{2}\left(r_{2} \kappa\right)_{x x}\right) \\
A_{3}=-J D^{-1}\left(\kappa a_{2}^{T}-a_{2} \kappa^{T}\right)
\end{gathered}
$$

together with the evolutions

$$
\begin{gathered}
\kappa_{t}=-\left(a_{2}\right)_{x}-J A_{3} J \kappa \\
\left(\kappa_{1}\right)_{t}=-\frac{1}{2}\left(r_{1}\right)_{x x x}+\left(\kappa_{1} r_{1}\right)_{x}+\left(\kappa_{2} r_{2}\right)_{x}+\kappa_{1}\left(r_{1}\right)_{x}+\kappa_{2}\left(r_{2}\right)_{x} \\
\left(\kappa_{2}\right)_{t}=\frac{1}{2}\left(r_{2}\right)_{x x x}-\left(\kappa_{1} r_{2}\right)_{x}-\kappa_{1}\left(r_{2}\right)_{x}+\left(\kappa_{2} r_{1}\right)_{x}+\kappa_{2}\left(r_{1}\right)_{x}-\frac{1}{2}\left(r_{2}\|\kappa\|^{2}\right)_{x}+\pi^{2}(z) \cdot \kappa .
\end{gathered}
$$

One can now take the limit as $\kappa \rightarrow 0$ in each one of the elements involved to check that such a limit exists and it defines an evolution on $k_{1}$ and $k_{2}$ as in the statement of the theorem. This concludes the proof of the theorem and this section.

\section{The case $G=O(2 m, 2 m)$ and a Decoupled system of KdV equations}

In [M4] the author classified all differential invariants of curves in the Grassmanian Lagrangian, or manifold of Lagrangian planes in $\mathbb{R}^{2 m}$. This space corresponds to the case $G=S p(2 m)$. She showed that the differential invariants in the $\mathfrak{g}_{1}$ component of the Serre-Frenet equations were the eigenvalues of what is called the Lagrangian Schwarzian derivative of a curve of Lagrangian planes. Those would be invariants of projective type. She also showed that, for a proper choice of constant cross section producing a moving frame, the geometric Poisson brackets can be restricted to the space of invariants of projective type to produce $n$-decoupled first and second KdV Hamiltonian structures. She showed that there exists an invariant evolution of Lagrangian planes whose flow, when the initial conditions are properly chosen, induces a decoupled system of $\mathrm{KdV}$ equations in the eigenvalues of the Lagrangian Schwarzian derivative of the flow.

In this section we will show that the case $G / H$ with $G=O(2 m, 2 m)$ has differential invariants of projective type very similar to those for curves of Lagrangian planes. They are indeed given by the eigenvalues of the skew-symmetric Schwarzian derivative of the flow. We will see how there is a critical difference with the Lagrangian Grassmannian produced by the appearance of differential invariants of fifth and higher order. This is the first example of a $|1|$-graded parabolic manifold where this situation is found (although not the first one for which higher order differential invariants are found since projective curves certainly have higher order differential invariants). It is unclear if a cross section depending on derivatives of differential invariants of third order can be used to find a moving frame producing the same 
situation as in the Lagrangian Grassmannian. The case $G=O(2 m+1,2 m+1)$ is fundamentally different in the fact that each $u(x) \in G / H$ has a one dimensional kernel that evolves with the curve. That makes the application of the moving frame method more complicated as we can see in this section.

5.1. Classification of differential invariants of curves in $O(2 m, 2 m) / H$. The space $O(2 m, 2 m) / H$ and the action of $O(2 m, 2 m)$ on the quotient can be described as follows:

as before, we can locally factor an element of $O(n, n)$ as $g=g_{1} g_{0} g_{-1}$ where

$$
\begin{gathered}
g_{1}(Z)=\left(\begin{array}{cc}
I+Z & -Z \\
Z & I-Z
\end{array}\right), g_{-1}(Y)=\left(\begin{array}{cc}
I+Y & Y \\
-Y & I-Y
\end{array}\right) \\
g_{0}(\Theta)=\left(\begin{array}{cc}
\frac{1}{2}\left(\Theta+\Theta^{-T}\right) & \frac{1}{2}\left(\Theta^{-T}-\Theta\right) \\
\frac{1}{2}\left(\Theta^{-T}-\Theta\right) & \frac{1}{2}\left(\Theta+\Theta^{-T}\right)
\end{array}\right),
\end{gathered}
$$

where $Z$ and $Y$ are skew-symmetric matrices and where $\Theta \in G L(n, \mathbb{R})$. This factorization is determined by the gradation (3.4) described in [Oc]. With this description $H=G_{1} \cdot G_{0}$, where $G_{1}$ is the subgbroup generated by elements of the form $g_{1}(Z)$ and $G_{0}$ is the one generated by elements of the form $g_{0}(\Theta)$. Thus, $G_{-1}$ can be chosen to be a section of $O(2 m, 2 m) / H$ and the relation $g g_{-1}(u)=$ $g_{-1}(g \cdot u) h$ for some $h \in H$ provides us with a formula for the action of $O(2 m, 2 m)$ on $O(2 m, 2 m) / H$. It is uniquely determined to be

$$
g \cdot u=\Theta(u+Y)\left(\Theta^{-T}+4 Z \Theta(u+Y)\right)^{-1} .
$$

The Lie algebra decomposition is given by $\mathfrak{g}=\mathfrak{g}_{1} \oplus \mathfrak{g}_{0} \oplus \mathfrak{g}_{-1}$, with $V_{i} \in \mathfrak{g}_{i}, i=$ $1,0,-1$, given by

$$
\begin{gathered}
V_{1}(z)=\left(\begin{array}{cc}
z & -z \\
z & -z
\end{array}\right), V_{-1}(y)=\left(\begin{array}{cc}
y & y \\
-y & -y
\end{array}\right) \\
V_{0}(A+B)=\left(\begin{array}{ll}
A & B \\
B & A
\end{array}\right)
\end{gathered}
$$

where $z, y, A$ are skew symmetric matrices, and $B$ is a symmetric matrix. Notice that $A$ and $B$ are the symmetric and skew-symmetric components of $C=A+B$. The commutation relations of the algebra are given by

$$
\begin{gathered}
{\left[V_{1}(z), V_{-1}(y)\right]=4 V_{0}(z y), \quad\left[V_{0}(C), V_{1}(z)\right]=V_{1}\left(C z+z C^{T}\right)} \\
{\left[V_{-1}(y), V_{0}(C)\right]=V_{-1}\left(y C+C^{T} y\right)}
\end{gathered}
$$

As before, we now proceed to write down normalization equations for this action and to use them to determine a moving frame along a curve. We are assuming $g=g_{1}(Z) g_{0}(\Theta) h_{-1}(Y)$. The zero order normalization equation is simply $g \cdot u=0$ which is readily solved choosing $Y=-u$. The first order normalization equations, after restricted to previous normalizations results in

$$
g \cdot u^{(1)}=\Theta u_{1} \Theta^{T}
$$

It is a well-known result in linear algebra that any generic skew symmetric matrix can be taken to the matrix

$$
J=\left(\begin{array}{cc}
0 & I_{m} \\
-I_{m} & 0
\end{array}\right)
$$


under a transformation (5.2). Thus, we choose the first normalization equation to be

$$
\Theta u_{1} \Theta^{T}=J
$$

which determines $\Theta$ up to an element of the symplectic group $\operatorname{Sp}(2 m)$.

Let $\Theta=\theta \mu$ for some $\theta \in \operatorname{Sp}(2 m)$ to be determined by later normalizations. As in our previous case, the second order normalization equations will determine the factor $\rho_{1}$ in the moving frame. Indeed, if we differentiate once more the action and substitute the values we have obtained in previous normalizations we obtain

$$
g \cdot u^{(2)}=\Theta u_{2} \Theta^{T}-8 J Z J=0 .
$$

This equation solves for $Z$ in terms of $\Theta$, which we still have to determine completely. That is

$$
Z=\frac{1}{8} J \Theta u_{2} \Theta^{T} J
$$

The third order normalization equations will determine part of the $\mathfrak{g}_{1}$ component of the Serre-Frenet equations. These equations, after some manipulation, are given by

$$
g \cdot u^{(3)}=\theta \mu\left(u_{3}-\frac{3}{2} u_{2} u_{1}^{-1} u_{2}\right) \mu^{T} \theta^{T} .
$$

We call

$$
S(u)=\mu\left(u_{3}-\frac{3}{2} u_{2} u_{1}^{-1} u_{2}\right) \mu^{T}
$$

a skew-symmetric Schwarzian derivative of $u$, unique up to the action of an element of the symplectic group. This is the skew-symmetric version of the Lagrangian Schwarzian derivative, first introduced by V. Ovsienko in [Ovs]. The question now is: which one is the normal form of a generic skew symmetric matrix under the above action of the symplectic group? A paper by Williamson ([Wi]) classifies normal forms in a way that can be used in our context. The following theorem is one of the results of Williamson's paper (in fact he gives earlier references for this particular case).

Theorem 12. Let $A$ and $B$ be two similar matrices. Assume $A H=H A^{T}$ and $B H=H B^{T}$. Then, the similarity matrix can be chosen such that $M H M^{T}=H$.

In the case at hand, if $S_{1}$ is a generic skew symmetric matrix with eigenvalues $\pm a_{k} i, k=1,2, \ldots, m$ and if

$$
S_{2}=\left(\begin{array}{cc}
0 & \mathbf{D} \\
-\mathbf{D} & 0
\end{array}\right)
$$

with $\mathbf{D}=\operatorname{diag}\left(a_{k}\right)$, then $A_{i}=S_{i} J$ holds $A_{i} J H=H\left(A_{i} J\right)^{T}$ for $H=J$. Furthermore, they are also similar (they have both double $a_{k}$ eigenvalues and they are diagonalizable) and so we can choose an element of the symplectic group as similarity element. That is

$$
g S_{1} J g^{-1}=S_{2} J
$$

where $g \in \operatorname{Sp}(2 m)$. On the other hand, if $g \in \operatorname{Sp}(2 m), g^{-1}=-J g^{T} J$ and hence we get

$$
g S_{1} g^{T}=S_{2}
$$

We just obtained the following Theorem. 
Theorem 13. Let $S$ be a nondegenerate $2 m \times 2 m$ skew-symmetric matrix and assume $\pm a_{k} i$ are its eigenvalues, $k=1,2, \ldots, m$. Then, there exists an element of $\operatorname{Sp}(2 m)$ such that

$$
\theta S \theta^{T}=\mathcal{D}=\left(\begin{array}{cc}
0 & \mathbf{D} \\
-\mathbf{D} & 0
\end{array}\right)
$$

where $\mathbf{D}$ is the diagonal matrix having $a_{k}$ down the diagonal. The element $\theta$ is unique up to an element of $\operatorname{Sp}(2 m)$ commuting with $\mathcal{D}$.

Theorem 14. The differential invariants of a generic curve in $O(2 m, 2 m) / H$ have all order three or higher. Let $\theta$ be chosen as in the previous theorem for $S=S(u)$ as in (5.7). Then, the entries of the matrix $\mathbf{D}$ generate all differential invariants of third order for $u$.

We call the entries of $\mathbf{D}$ the differential invariants of projective type for curves in $O(2 m, 2 m) / H$.

Let's now return to the remaining part of the moving frame that needs to be determined. The symplectic group is itself semisimple and has a local factorization of the form

$$
\left(\begin{array}{ll}
I & z \\
0 & I
\end{array}\right)\left(\begin{array}{cc}
g & 0 \\
0 & g^{-T}
\end{array}\right)\left(\begin{array}{ll}
I & 0 \\
y & I
\end{array}\right)
$$

where $z$ and $y$ are symmetric matrices, and where $g \in \mathrm{GL}(m)$. It is trivial to check that elements commuting with $\mathcal{D}$ correspond to those with a factorization of the form

$$
d=\left(\begin{array}{cc}
I & \mathbf{D}_{1} \\
0 & I
\end{array}\right)\left(\begin{array}{cc}
\mathbf{D}_{2} & 0 \\
0 & \mathbf{D}_{2}^{-T}
\end{array}\right)\left(\begin{array}{cc}
I & 0 \\
\mathbf{D}_{3} & I
\end{array}\right)
$$

where the three matrices $\mathbf{D}_{i}, i=1,2,3$ are diagonal. They are the part of the moving frame still to be determined. This time we need to move to the fourth order normalization equations. They can be written as

$$
\left.g \cdot u^{(4)}\right|_{\mathcal{N}}=d \theta \mu\left(u_{4}-2\left(u_{3} u_{1}^{-1} u_{2}+u_{2} u_{1}^{-1} u_{3}\right)+3 u_{2} u_{1}^{-1} u_{2} u_{1}^{-1} u_{2}\right) \mu^{T} \theta^{T} d^{T}
$$

We will show how, if $m>3, d$ can be determined from $3 m$ normalization equations chosen from the entries of the matrix above. If $m \leq 3$ further normalizations are needed. Let's call

$$
\left(\begin{array}{cc}
N_{1} & N_{3} \\
-N_{3} & N_{2}
\end{array}\right)=\theta \mu\left(u_{4}-2\left(u_{3} u_{1}^{-1} u_{2}+u_{2} u_{1}^{-1} u_{3}\right)+3 u_{2} u_{1}^{-1} u_{2} u_{1}^{-1} u_{2}\right) \mu^{T} \theta^{T}
$$

Where $N_{1}$ and $N_{2}$ are skew-symmetric and $N_{3} \in \operatorname{gl}(m)$. From Fels and Olver's recurrence formulas ([FO2]) we know that the diagonal of $N_{3}$ is given by $\mathbf{D}_{x}$. Hence, we can only normalize outside the diagonals.

It is not hard to see that, if $m>3$, a total of $3 m$ normalizations can be performed in (5.9). That means we will have $3 m$ fifth order differential invariants appearing in the positions of $\rho \cdot u^{(5)}$ corresponding to the normalized positions chosen in $\rho \cdot u^{(4)}$. This is, we obtain $m$ third order invariants, $2 m(m-2)$ fourth order invariants and $3 m$ fifth order invariants.

If $m \leq 3$ one needs to go higher to normalize entries in the fifth order normalization equations. In those cases we also obtain sixth order invariants corresponding to the normalized fifth order entries in $\rho \cdot u^{(5)}$ as located in $\rho \cdot u^{(6)}$. For $m=1$ we are in the $\mathbb{R P}^{1}$ case. For $m=2$ one can check that we have two differential invariants of projective type, two of fifth order and two of sixth order. For $m=3$ one has three third order differential invariants, four fourth order ones, seven fifth 
order and one sixth order. This is not obvious, in fact one can show that the rank of the twelve equations for the nine unknown is eight. The author used MAPLE to check this.

We don't need more details for the calculations that follow in this section, but there are many important points regarding the fourth normalization that are essential for our next section. Indeed, the choice of fourth normalization constants (and hence fourth and fifth order invariants) are key to the study of integrable level sets of some geometric realizations. Therefore, we will return to exactly this point shortly. The next theorem describes the Serre-Frenet frame associated to this moving frame.

Theorem 15. Let $u$ be a generic curve in $O(2 m, 2 m) / H$. Let $\rho$ be a moving frame determined as above. Then, the (left-invariant) Serret-Frenet equations associated to $\rho$ are given by $\rho\left(\rho^{-1}\right)_{x}=-\rho_{x} \rho^{-1}=K$, with $K$ equals

$$
\begin{gathered}
K=\left(\begin{array}{cc}
J & J \\
-J & -J
\end{array}\right)+\frac{1}{8}\left(\begin{array}{ll}
\mathcal{D} & -\mathcal{D} \\
\mathcal{D} & -\mathcal{D}
\end{array}\right)+\frac{1}{2}\left(\begin{array}{cc}
R-R^{T} & R+R^{T} \\
R+R^{T} & R-R^{T}
\end{array}\right) \\
=V_{-1}(J)+\frac{1}{8} V_{1}(\mathcal{D})+V_{0}(R)
\end{gathered}
$$

where $R$ is of the form

$$
R=\left(\begin{array}{cc}
R_{1} & R_{2} \\
R_{3} & -R_{1}^{T}
\end{array}\right) \in \operatorname{Sp}(2 m)
$$

with $R_{2}$ and $R_{3}$ symmetric. The matrix $R$ contains in the entries off the diagonals of $R_{i}, i=1,2,3$, a generating set of independent fourth order differential invariants. The diagonals of $R_{i}, i=1,2,3$ contain a set of $3 \mathrm{~m}$ independent and generating differential invariants of order 5 for $m>3$ and of order 5 and higher if $m \leq 3$.

Proof. As in the previous chapter we need to calculate the Serre-Frenet equations. We will skip all straightforward calculations and just give the result. If $\rho$ is as above, one can check that $\rho_{1} \rho_{0} \rho_{-1}\left(\rho_{-1}^{-1}\right)_{x} \rho_{0}^{-1} \rho_{1}^{-1}$ is given by

$$
\begin{gathered}
\left(\begin{array}{cc}
J & J \\
-J & -J
\end{array}\right)+2\left(\begin{array}{cc}
Z J-J Z & Z J+J Z \\
Z J+J Z & Z J-J Z
\end{array}\right)+4\left(\begin{array}{cc}
-Z J Z & Z J Z \\
-Z J Z & Z J Z
\end{array}\right) \\
=V_{-1}(J)+4 V_{0}(Z J)-4 V_{1}(Z J Z) .
\end{gathered}
$$

Similarly, if $\Gamma=\Theta_{x} \Theta^{-1}$, then $\rho_{1} \rho_{0}\left(\rho_{0}^{-1}\right)_{x} \rho_{1}^{-1}$ is given by

$$
V_{0}\left(\Gamma^{T}\right)+V_{1}\left(-Z \Gamma+(Z \Gamma)^{T}\right) .
$$

and $\rho_{1}\left(\rho_{1}^{-1}\right)_{x}$ is given by

$$
-V_{1}\left(Z_{x}\right)
$$

Clearly, if $K=K_{-1}+K_{0}+K_{1}, K_{i} \in \mathfrak{g}_{i}$, then $K_{-1}$ is as in the statement of the Theorem. Also, differentiating the first and second order normalization equations one can show that

$$
Z_{x}+4 Z J Z+Z \Gamma-(Z \Gamma)^{T}=\frac{1}{8} J d \theta S(u) \theta^{T} d^{T} J=\frac{1}{8} J \mathcal{D} J=-\frac{1}{8} \mathcal{D} .
$$

This gives the value of $K_{1}$ that the statement of the Theorem shows. Finally, let $R=\frac{1}{2}\left(\Gamma^{T}+J \Gamma J\right)$ (that is, $R$ is the symplectic part of $\Gamma^{T}$ ). Since $R^{T}=J R J$, this 
matrix will be of the form

$$
R=\left(\begin{array}{cc}
R_{1} & R_{2} \\
R_{3} & -R_{1}^{T}
\end{array}\right) \in \mathrm{Sp}(2 m)
$$

with $R_{2}$ and $R_{3}$ symmetric. Assume the fourth order normalization equation, after being normalized, is given by

$$
\rho \cdot u^{(4)}=I_{4}=\left(\begin{array}{cc}
M_{1} & M_{3} \\
-M_{3}^{T} & M_{2}
\end{array}\right)
$$

with $M_{1}$ and $M_{2}$ skew-symmetric. Then, differentiating the third order normalization equations and using the fourth order one we get the relation

$$
\mathcal{D}_{x}-I_{4}=R^{T} \mathcal{D}+\mathcal{D} R .
$$

Notice that this is equivalent to

$$
[R, J \mathcal{D}]=J\left(I_{4}-\mathcal{D}_{x}\right) .
$$

This results in $\left[\mathbf{D}, R_{2}\right]=-2 M_{1},\left[R_{3}, \mathbf{D}\right]=-2 M_{2}$ and $\left[R_{1}, \mathbf{D}\right]=2\left(\mathbf{D}_{x}-M_{3}\right)$. We know from Fels and Olver's recurrence formulas that $M_{3}$ has in its diagonal $\mathbf{D}_{x}$. Therefore, the matrix $R$ has off its diagonals all the entries of $I_{4}$, the fourth order independent and generating differential invariants. Finally, there are only $3 \mathrm{~m}$ entries in $K$ that have not been determined to be a differential invariant of order less or equal to four (constant or otherwise). These are the diagonal entries of $R_{i}$, $i=1,2,3$ (we will call these the block diagonals). The study in $[\mathrm{H}]$ shows that the Frenet-Serre equations contain in its entries a generating and independent set of differential invariants. Hence, generators of the $3 \mathrm{~m}$ higher order invariants need to be placed along those three diagonals. This concludes the proof of the theorem.

Our next subsection will study the evolution of $\mathcal{D}$. It is well known that the KdV-Schwarzian evolution for $u(t, x): \mathbb{R}^{2} \rightarrow \mathbb{R P}^{1}$ given by

$$
u_{t}=u_{3}-\frac{3}{2} \frac{u_{2}^{2}}{u_{1}}=u_{1} S(u)
$$

is invariant under the projective action of $P S L(2 . \mathbb{R})$. The induced evolution on the generating projective invariant, the Schwarzian derivative $S(u)=\frac{u_{3}}{u_{1}}-\frac{3}{2} \frac{u_{2}^{2}}{u_{1}^{2}}$, is a KdV evolution. That is

$$
S(u)_{t}=S(u)_{3}+3 S(u)_{2} S(u)_{1} .
$$

Because the projective-type invariants of KdV-type are eigenvalues of the Schwarzian derivative, one would expect to have an evolution of decoupled KdV equations as a level set of the evolution induced on the differential invariants by the skewsymmetric equivalent of the KdV-Schwarzian evolution for the flow $u(t, x)$. Such was the case for the Lagrangian Grassmannian. Indeed, in that case, the Lagrangian $\mathrm{KdV}$-Schwarzian evolution was given by (the minus comes from using right invariant frames here)

$$
-\left(\rho_{-1}\right)_{x} \rho_{-1}^{-1}=\operatorname{Ad}\left(\rho_{0}^{-1}\right)(\widehat{\mathcal{D}})
$$

where $\widehat{\mathcal{D}}$ is the equivalent to $\mathcal{D}$ here, namely the eigenvalues of the Lagrangian Schwarzian derivative of the flow (a symmetric functional matrix). In [M4] the author showed that the corresponding evolution of the differential invariants of the flow has as a level set the submanifold of curves for which the fourth order 
differential invariants vanish (there were no higher order invariants in this case). Furthermore, the induced evolution on $\mathcal{D}$ when initial conditions are chosen in that level set is a decoupled system of $\mathrm{KdV}$ equations. Indeed, the geometric Poisson brackets reduced to the level set to produce a decoupled system of biHamiltonian structures for $\mathrm{KdV}$, even though only one of them reduced to the complete manifold of differential invariants.

Our next subsection will show that the appearance of fifth order differential invariants changes considerably the picture. We will show how no choice of constant (or even zeroth order on $\mathcal{D}$ ) fourth order cross section will result in a completely integrable level set of this type. In fact, none of these choices of cross sections will result on a reduced biHamiltonian structure to the set of projective-type differential invariants. On the other hand, as fourth order differential invariants vanish, some conditions on a constant cross section guarantee that third and fifth order differential invariants decouple and $\mathcal{D}$ evolve following a decoupled system of $\mathrm{KdV}$ equations. This is the first example for which this happens. It is unclear whether or not a choice of section depending on derivatives of $\mathcal{D}$ will allow us to prove the existence of a level set for vanishing fourth and fifth order. The method presented here makes the calculations of that study too complicated. It is perhaps best to learn how the geometry of $|1|$-graded parabolic manifolds generates these completely integrable level sets. Once that is well understood we will be able to understand why, and to what extent, this case is different from the Lagrangian Grassmannian.

5.2. Decoupled KdV equations associated to $O(2 m, 2 m) / H$. Let us assume that

$$
u_{t}=F\left(\mathbf{k}, \mathbf{k}_{1}, \mathbf{k}_{2}, \ldots\right)
$$

is an evolution of curves in $G / H$, invariant under the action of the group $G$ where $G=O(2 m, 2 m)$, and where $\mathbf{k}$ represents the vector of differential invariants, that is, of independent entries in $\mathcal{D}$ and $R$. It was shown in [M1] that the induced evolution on $\mathbf{k}$ could be written, in terms of a right invariant moving frame, as

$$
\rho_{-1}\left(\rho_{-1}^{-1}\right)_{t}=\mathrm{Ad}\left(\rho_{0}^{-1}\right) \beta
$$

whenever $\rho_{-1}$ could be identified with $-u$ the way we did here, and for any $\beta\left(\mathbf{k}, \mathbf{k}_{1}, \ldots\right) \in \mathfrak{g}_{-1}$ with differential invariant entries. This is true for any $|1|$ graded parabolic manifold. Again, the slightly different formulation is merely due to $\rho$ being a right invariant moving frame while the one in [M1] is a left invariant moving frame. Let $\Theta$ be determined by our moving frame $\rho$.

Theorem 16. Let us have a flow of curves in $G / H, G=O(2 m, 2 m)$, evolve following any given invariant evolution. Then the evolution can be written as

$$
u_{t}=\Theta^{-1} v \Theta^{-T}
$$

where $v=v\left(\mathbf{k}, \mathbf{k}_{x x}, \ldots\right)$ is any invariant skew-symmetric matrix.

This Theorem is a direct consequence of the relation (5.17) when applied to our moving frame.

In the Lagrangian Grassmanian case, evolutions defined by a diagonal matrix $v$ preserved the level set where fourth order differential invariants vanish. That is, if the initial condition was chosen to be a curve with vanishing fourth order 
differential invariants, then the entire flow will have vanishing differential invariants. Furthermore, if $v=\widehat{\mathcal{D}}$, then the evolution of curves was

$$
\widehat{u}_{t}=\widehat{u}_{3}-\frac{3}{2} \widehat{u}_{2} \widehat{u}_{1}^{-1} \widehat{u}_{2}
$$

and this was called the Lagrangian Schwarzian KdV evolution. This evolution induced a decoupled system of $\mathrm{KdV}$ equations on $\widehat{\mathcal{D}}$. Here $\widehat{u}$ is a flow of Lagrangian planes in $\mathbb{R P}^{2 n}$, represented as a flow of symmetric matrices (see [M4]). It is thus natural to ask whether or not the corresponding skew symmetric evolution

$$
u_{t}=\Theta^{-1} \mathcal{D} \Theta^{-T}=u_{3}-\frac{3}{2} u_{2} u_{1}^{-1} u_{2}
$$

where $u(t, x)$ is a flow of skew symmetric matrices, preserves the manifold of vanishing differential invariants not of projective type. The answer is that, regardless of the choice of constant cross section, the manifold of vanishing fifth order differential invariants is never preserved. The manifold of vanishing fourth order differential invariants is preserved only under some conditions of the cross section, conditions that are easily satisfied in high dimensions. In this case the evolutions of third and fifth order invariants decouple, and the third order invariants evolve following a decoupled system of $\mathrm{KdV}$ equations.

Before stating any Theorem we will do some calculations and obtain some formulas. Recall that given any matrix we can split it into symplectic and non-symplectic components as

$$
A=\frac{1}{2}\left(A+J A^{T} J\right)+\frac{1}{2}\left(A-J A^{T} J\right)=A^{S}+A^{m} .
$$

We call $\mathfrak{m}$ the algebra of all non-symplectic components, i.e., matrices $A$ such that $A J-J A^{T}=0$. Clearly $\mathfrak{m}$ is a complement to $\operatorname{sp}(2 m)$ in $\mathrm{gl}(2 m)$. We will also split any matrix $A$ as

$$
A=A^{d}+A^{n d}
$$

where $A^{d}$ has non-zero entries only along block diagonals, and $A^{n d}$ has zero entries in its block diagonals.

Lemma 2. Let $\rho$ be the moving frame given in the previous subsection along $u(t, x)$, a flow solution of (5.19). Let $N=-\rho_{t} \rho^{-1}=\rho\left(\rho^{-1}\right)_{t}$ and let $N=N_{-1}+N_{0}+N_{1}$ according to the gradation, with $N_{i}=V_{i}\left(n_{i}\right), i=-1,0,1$. Then

$$
\begin{aligned}
n_{-1} & =\mathcal{D} \\
8 n_{1} J & =\left(n_{-1}\right)_{x x} J+\left[R, n_{-1} J\right]_{x}+\left[R,\left(n_{-1}\right)_{x} J\right]+\left[R,\left[R, n_{-1} J\right]\right]+\mathcal{D} n_{-1}
\end{aligned}
$$

and $n_{0}=n_{0}^{S}+n_{0}^{m}$ splits into symplectic and non-symplectic parts, with

$$
\begin{aligned}
n_{0}^{m} & =\frac{1}{2}\left(n_{-1}\right)_{x} J+\frac{1}{2}\left[R, n_{-1} J\right] \\
{\left[n_{0}^{S}, \mathcal{D} J\right] } & =\left[R_{x x},\left(n_{-1}\right)\right]+3\left[R_{x},\left(n_{-1}\right)_{x} J\right]+3\left[R,\left(n_{-1}\right)_{x x} J\right]+\left[n_{-1} J,\left[R, R_{x}\right]\right] \\
& +3\left[R,\left[R, n_{-1} J\right]_{x}\right]^{n d}+\left[R,\left[R,\left[R, n_{-1} J\right]\right]\right]^{n d}+\frac{3}{2}\left[R, \mathcal{D} n_{-1}\right] .
\end{aligned}
$$

Proof. The proof of this lemma is rather simple and has calculations very similar to those in the proof of section 4.2. Hence we will avoid unnecessary details. If we apply the structure equation for the (left invariant) Maurer-Cartan form of 
$O(2 m, 2 m)$ to the vector fields defined by $\rho_{x}$ and $\rho_{t}$ along $\rho$ we get the relationship (see $[\mathrm{M} 1])$

$$
K_{t}=N_{x}+[K, N]
$$

where $K=\rho\left(\rho^{-1}\right)_{x}$ and $N=\rho\left(\rho^{-1}\right)_{t}$. In [M1] it was shown that, if $N=N_{1}+$ $N_{0}+N_{-1}$ according to the gradation, then $N_{-1}=V_{-1}(v)$, where $v$ is given by the curve evolution (5.18). That is, in our case $v=\mathcal{D}=n_{-1}$

Using calculations similar to those in the proof of the previous section, we can obtain the equations above. In fact, we get

$$
\begin{aligned}
0 & =N_{-1}+\left[K_{-1}, N_{0}\right]+\left[K_{0}, N_{-1}\right] \\
\left(K_{0}\right)_{t} & =\left(N_{0}\right)_{x}+\left[K_{0}, N_{0}\right]+\left[K_{-1}, N_{1}\right]+\left[K_{1}, N_{-1}\right] \\
\left(K_{1}\right)_{t} & =\left(N_{1}\right)_{x}+\left[K_{1}, N_{0}\right]+\left[K_{0}, N_{1}\right] .
\end{aligned}
$$

After we substitute the representatives of each graded component, apply the commutation relations of the Lie algebra and split symplectic and non-symplectic components in $\mathfrak{g}_{0}$, these equations become

$$
\begin{aligned}
0 & =\left(n_{-1}\right)_{x}+2 J n_{0}^{m}-J\left[R, n_{-1} J\right] \\
R_{t} & =\left(n_{0}^{S}\right)_{x}+\left[R, n_{0}^{S}\right] \\
0 & =\left(n_{0}^{m}\right)_{x}+\left[R, n_{0}^{m}\right]-4 n_{1} J+\frac{1}{2} \mathcal{D} n_{-1} \\
\mathcal{D}_{t} & =8\left(n_{1}\right)_{x}-\left(n_{0} \mathcal{D}+\mathcal{D} n_{0}^{T}\right)-\left[R, n_{1} J\right] J .
\end{aligned}
$$

The first equation solves for $n_{0}^{m}$ in terms of $n_{-1}=\mathcal{D}$. The third equation solves for $n_{1}$ in terms of $n_{0}^{m}$ and $n_{-1}$. When we substitute these values in the last equation, and apply Jacobi's identity, we can obtain the formula shown for $\left[n_{0}^{S}, \mathcal{D} J\right]$ in the statement of the lemma. Notice that $\left[n_{-1} J,\left[R, R_{x}\right]\right]$ has zero block diagonal entries.

This lemma additionally tells us what the evolution for $\mathcal{D}$ is. We have, after some rewriting

$$
\begin{aligned}
\mathcal{D}_{t} J & =\left(n_{-1}\right)_{x x x} J+\left(\mathcal{D} n_{-1}\right)_{x}+\mathcal{D}\left(n_{-1}\right)_{x} \\
& +3\left[R,\left[R, n_{-1} J\right]_{x}\right]^{d}+\left[R,\left[R,\left[R, n_{-1} J\right]\right]\right]^{d} .
\end{aligned}
$$

One can easily recognize the portion of the evolution involving no $R$ as a decoupled system of KdV equations. This system is, in general, perturbed by terms depending on higher order invariants.

Notice also that the lemma determines only the entries of $n_{0}^{S}$ outside its block diagonals. Indeed, $\left[n_{0}^{S}, \mathcal{D} J\right]$ has always zero diagonals in its four blocks (it is quite clear that, for a generic curve, the map $\operatorname{ad}(\mathcal{D} J)$ is one to one when we omit block diagonals from its domain). Therefore, we still have to determine the diagonals of $n_{0}^{S}$. Because these diagonals determine the evolution of the higher order differential invariants and this is the crucial difference with the Lagrangian Grassmannian case we will write it out in a separate lemma. Let us denote by $\pi: S p(2 m) \rightarrow \mathbb{R}^{3 m}$ the map projecting a matrix on its $3 m$ normalization entries. That is, the entries that were normalized in the fourth order normalization equations when we apply $a d(D)$ to the symplectic matrix and multiply the relation (5.16) by $J$ on the left. Notice that, if $S$ is symmetric, then both $S J$ and $J S$ are symplectic, if $M$ is skew symmetric, then $J M$ and $M J$ are in $\mathfrak{m}$, where $\mathfrak{m}$ is the complement of the symplectic algebra in $\operatorname{gl}(2 m)$ (that is, the set of non-symplectic components), and that if $R$ is symplectic then $\operatorname{ad}(D)(R) \in \mathfrak{m}$. That implies that, if $I_{4}$ is skew-symmetric and 
has a number of normalized entries, then $J I_{4}$ is in $\mathfrak{m}$ and it has the corresponding normalized entries assigned in a one-to-one fashion since they are off-diagonals. Since $\operatorname{ad}(\mathcal{D} J)(R)$ is also in $\mathfrak{m}$, a number of its off-diagonal entries are normalized, exactly $3 m$ of them. Since $a d(\mathcal{D} J)$ is one-to-one off the diagonals this determine $3 m$ normalized entries in $R$. Those are the ones defining $\pi$. For simplicity we will also denote by $\pi$ the projection of normalized entries found in skew-symmetric matrices, that is, the map projecting on those entries that were normalized in $I_{4}$.

Lemma 3. The equation

$$
\pi\left(R_{t}\right)=\pi\left(\left(n_{0}^{S}\right)_{x}+\left[R, n_{0}^{S}\right]\right)
$$

determines the block diagonals of $n_{0}^{S}$.

Notice that $\pi(R)$ will depend on third order differential invariants.

Proof. The proof is based on the fourth normalization equations. Let $\tilde{I}_{4}$ be defined by $\tilde{d} \tilde{I}_{4} \tilde{d}^{T}=I_{4}$, where $\tilde{d}$ is the component of the moving frame that was determined last using the fourth normalization equations. Notice that the set of matrices $d$ form a subgroup of $\operatorname{Sp}(2 m)$, let us call it $G_{d}$ and its Lie-algebra $\mathfrak{d}$ is given by matrices of the form

$$
\left(\begin{array}{cc}
a & b \\
c & -a
\end{array}\right)
$$

where all $a, b, c$ are diagonal matrices. Since the map $G_{d} \rightarrow \mathbb{R}^{3 m}$ given by $d \rightarrow$ $\pi\left(d \tilde{I}_{4} d^{T}\right)$ has full rank equal $3 m$ (the entries and normalization constants where chosen so we could solve for $\tilde{d}$ ), its differential at $\tilde{d} \in G_{d}$ will also have full rank. The differential is given by

$$
A \rightarrow \pi\left(A \tilde{d} \tilde{I}_{4} \tilde{d}^{T}+\tilde{d} \tilde{I}_{4} \tilde{d}^{T} A^{T}\right)=\pi\left(A I_{4}+I_{4} A^{T}\right)
$$

for any $A \in \mathfrak{d}$. Using relation (5.16) we can rewrite this as

$$
A \rightarrow \pi\left(A J[\mathcal{D} J, R]+J[\mathcal{D} J, R] A^{T}\right)
$$

since $\pi\left(A \mathcal{D}_{x}+\mathcal{D}_{x} A^{T}\right)=0$. The above can be rewritten as

$$
A \rightarrow-J\left[A^{T},[J \mathcal{D}, R]\right]
$$

which proves the Lemma since the normalized entries are not in block diagonal positions.

Let us call $n_{0}^{d}$ the block diagonal components of $n_{0}^{S}$. Here we can immediately see one of the main differences with the Lagrangian case. One can make normalized entries in $R$ vanish when fourth oder differential invariants vanish (that was the case for the Lagrangian Grassmannian). This can be achieved in the normalization process by choosing a cross section for which entries in $d \tilde{I}_{4} d^{T}$ are made equal to each other in order to solve for $d$. Thus, all entries depend on fourth and higher order differential invariants and will vanish when they vanish. But in that case, as we approach the set of vanishing higher order invariants $n_{0}^{d}$ will blow up, and with it the time evolution of the higher order differential invariants. This is the essence of the proof of the following Theorem.

Theorem 17. Let $R$ be defined using any fourth order cross section which is constant or zeroth order in $\mathcal{D}$. That is, $\pi\left(c_{4}\right)$ are either constant or functions of $\mathcal{D}$. Then, the set of vanishing fifth or higher order differential invariants is not preserved by the skew-symmetric Schwarzian KdV evolution. 
Proof. The evolution of fifth and higher differential invariants is defined by the block diagonal components of the equation

$$
R_{t}=\left(n_{0}^{S}\right)_{x}+\left[R, n_{0}^{S}\right]
$$

where $n_{0}^{S}$ is determined outside its block diagonals by Lemma 2 and $n_{0}^{d}$ is determined by Lemma 3. If $\pi\left(c_{4}\right)$ is either constant or zeroth order, then, according to both lemmas, the highest order, non vanishing terms in $\mathcal{D}$ found outside the diagonals of $n_{0}^{S}$ are order 2 and the higher order term in the block diagonals is order three (normalized entries in $R$ are functions of $\mathcal{D}$ and $\mathcal{D}_{t}$ is third order).

The highest order term in $\mathcal{D}$ in the block diagonals of $R_{t}$ is given by the highest order term in $\left(n_{0}^{d}\right)_{x}$ since this term has third and fourth order terms, while the block diagonals of $\left[R, n_{0}^{S}\right]$ have order two only. The third and fourth order terms in $\left(n_{0}^{d}\right)_{x}$ are found using the relation (5.21) in Lemma 3. Indeed, if we denote by 4 the fourth order term, we get that

$$
\pi\left(R_{x t}\right)_{4}=\pi\left(\left(n_{0}^{S}\right)_{x x}+\left[R,\left(n_{0}^{d}\right)_{x}\right]\right)_{4} .
$$

If we apply now $a d(\mathcal{D} J)$, which is one-to-one outside block diagonals, we get that

$$
\pi\left(\left[R_{x t}, \mathcal{D} J\right]+\left[R_{x}, \mathcal{D}_{t} J\right]+\left[R_{t}, \mathcal{D}_{x} J\right]+\left[R, \mathcal{D}_{x t}\right]\right)=\pi([R, \mathcal{D} J])
$$

which is either zero or first order in $\mathcal{D}$. Therefore, the fourth order term in $\left[R_{x t}, \mathcal{D} J\right]$ is given by $-\pi\left[R, \mathcal{D}_{x x x x} J\right]$.

From the formula for $n_{0}^{S}$ in Lemma 2 we see that

$$
\pi\left[\left(n_{0}^{S}\right)_{x x}, \mathcal{D} J\right]_{4}=2\left[\left[R,\left(n_{-1}\right)_{x x x x} J\right]=2\left[R, \mathcal{D}_{x x x x} J\right] .\right.
$$

Therefore, the highest order term in $\pi\left[\left[R,\left(n_{0}^{d}\right)_{x}\right], \mathcal{D} J\right]$ is $-3 \pi\left[R,\left(n_{-1}\right)_{x x x x} J\right]$. This will generically vanish only if $\pi(R)=0$. This is not possible since in this case $n_{0}^{d}$ will blow up (besides, the fourth order normalization equations would not be full rank in this submanifold).

Finally, we will show that, under some conditions on $\pi(R)$, the submanifold of vanishing fourth order invariants is preserved by the skew symmetric $\mathrm{KdV}$ Schwarzian evolutions, and that the evolution of third order invariants decouple from the fifth order, with $\mathcal{D}$ evolving following a decoupled system of KdV equations. We are assuming below that $m>3$, so we have only fifth order invariants.

Theorem 18. Let $\pi([R, \mathcal{D} J])=c_{4}$ be constant and assume that, as the fourth order invariants vanish, $[R, \widehat{R}]=\widehat{\widehat{R}}+$ block diagonals where $R, \widehat{R}$ and $\widehat{\widehat{R}}$ have non-zero entries in the same position (in the nonzero normalized positions). Assume also that $[R,[R, \widehat{R}]]^{d}=0$ for $\widehat{R}$ as above.

Then, if we choose initial conditions with vanishing fourth order invariants, these remain zero, $\mathcal{D}_{t}$ and $\left(R^{d}\right)_{t}$ decouple, and

$$
\mathcal{D}_{t} J=\mathcal{D}_{x x x} J+\left(\mathcal{D}^{2}\right)_{x}+\mathcal{D} \mathcal{D}_{x}
$$

a decoupled system of KdV equations.

Proof. Again we use here the two lemmas in this section. Since

$$
R_{t}=\left(n_{0}^{S}\right)_{x}+\left[R, n_{0}^{S}\right]
$$

we have that, if $\hat{\pi}(R)$ denotes the projection on non-diagonal and non-normalized entries, then the evolution of fourth order invariants is given by $\hat{\pi}\left(R_{t}\right)$. From Lemma 2 , if we have vanishing fourth order invariants, then $\hat{\pi}\left(\left[n_{0}^{S}, \mathcal{D} J\right]\right)=\left[\hat{\pi}\left(n_{0}^{S}\right), \mathcal{D} J\right]=0$ 
and hence $\hat{\pi}\left(n_{0}^{S}\right)=\hat{\pi}\left(n_{0}^{S}\right)_{x}=0$. Similarly we see that, away from diagonals $n_{0}^{S}$ has only non-vanishing entries in the normalized directions, and hence $\hat{\pi}\left(\left[R, n_{0}^{S}\right]\right)=0$. From here we see that if initial conditions are chosen such that their fourth order invariants vanish, the same condition will hold true for the entire flow.

Finally,

$$
\begin{aligned}
\mathcal{D}_{t} J & =\left(n_{-1}\right)_{x x x} J+\left(\mathcal{D} n_{-1}\right)_{x}+\mathcal{D}\left(n_{-1}\right)_{x} \\
& +3\left[R,\left[R, n_{-1} J\right]_{x}\right]^{d}+\left[R,\left[R,\left[R, n_{-1} J\right]\right]\right]^{d} .
\end{aligned}
$$

and, if $c_{4}$ is constant, $\left[R, n_{-1} J\right]_{x}=0$. Furthermore, under our assumptions $\left[R,\left[R,\left[R, n_{-1} J\right]\right]\right]^{d}=0$ and so the Theorem is proved.

Conditions $[R, \widehat{R}]=\widehat{\widehat{R}}+$ diagonals and $[R,[R, \widehat{R}]]^{d}=0$ are easily accomplished as the dimension grows. Indeed, as the fourth order differential invariants vanish, the number of nonzero entries in $R$ is always less or equal to $3 m$, while the number of available equations is $m(2 m-1)$. Thus, if $m$ is large enough (greater than 4 ) accomplishing the conditions get easier and easier. It is not clear if they hold for $m=4$. Some more details need further study: can we choose a first order transverse section in $\mathcal{D}$ to make the submanifold of fifth order vanishing invariants a level set of the evolution? What exactly happens in the Hamiltonian picture? Are fifth order invariants also of projective type in some sense? A better path is to study how the geometry of these manifolds generate the invariants and the evolutions. Research in that direction is underway.

Acknowledgements: This paper was written under the support of a grant from the Netherlandse Organisatie voor Wetenschappelijk Onderzoek (NWO). The author thanks the Vrije Universiteit in Amsterdam for a very pleasant visit during 20052006 .

\section{REFERENCES}

[C] E. Cartan. La Méthode du Repère Mobile, la Théorie des Groupes Continus et les Espaces Généralisés, Exposés de Géométrie 5, Hermann, Paris, 1935.

[Ca] A. Calini. Recent developments in integrable curve dynamics, in Geometric approaches to differential equations (Canberra 1995), pp 56-99. Cambridge University Press, Cambridge 2000.

[Fi] A. Fialkow. The Conformal Theory or Curves, Transactions of the AMS, 51; pp 435-568, 1942.

[FO1] M. Fels, P.J. Olver. Moving coframes. I. A practical algorithm, Acta Appl. Math., pp 99136, 1997.

[FO2] M. Fels, P.J. Olver. Moving coframes. II. Regularization and theoretical foundations. Acta Appl. Math., pp 127-208, 1999.

[G] M. L. Green. The moving frame, differential invariants and rigidity theorems for curves in homogeneous spaces, Duke Mathematical Journal, 45(4):735-779, 1978.

[Gr] P.A. Griffiths. On Cartan's method of Lie groups and moving frames as applied to uniqueness and existence questions in Differential Geometry, Duke Mathematical Journal, 41:775$814,1974$.

[Ha] R. Hasimoto. A soliton on a vortex filament, J. Fluid Mechanics, 51:477-485, 1972.

$[\mathrm{H}] \quad$ E. Hubert. Generation properties of differential invariants in the moving frame methods, in preparation.

[K] S. Kobayashi. Transformation Groups in Differential Geometry, Classics in Mathematics, Springer-Verlag, New York, 1972.

[KO] S. Kobayashi, T. Nagano. On filtered Lie Algebras and Geometric Structures I, Journal of Mathematics and Mechanics, 13(5):875-907, 1964. 
[M1] G. Marí Beffa. Geometric Poisson brackets in flat semisimple homogenous spaces, accepted for publication in the Asian Journal of Mathematics.

[M2] G. Marí Beffa. Poisson brackets associated to the conformal geometry of curves, Trans. Amer. Math. Soc. 357 (2005) 2799-2827.

[M3] G. Marí Beffa. Poisson Geometry of differential invariants of curves in nonsemisimple homogeneous spaces, Proc. Amer. Math. Soc. 134 (2006) 779-791.

[M4] G. Marí Beffa. On completely integrable geometric evolutions of curves of Lagrangian planes, accepted for publication in the Proceedings of the Royal academy of Edinburg, 2006.

[O] P.J. Olver. Equivalence, Invariance and Symmetry, Cambridge University Press, Cambridge, UK, 1995.

[O2] P.J. Olver. Moving frames and singularities of prolonged group actions, Selecta Math, 6: 41-77 (2000).

[OW] P.J. Olver, J.P. Wang. Classification of one-component systems on associative algebras, Proc. London Math. Soc., 81: 566-586 (2000).

[Oc] T. Ochiai. Geometry associated with semisimple flat homogeneous spaces, Transactions of the AMS, 152:pp 159-193, 1970.

[Ov] L.V. Ovsiannikov. Group Analysis of Differential Equations. Academic Press, New York (1982).

[Ovs] V. Ovsienko. Lagrange Schwarzian derivative and symplectic Sturm theory. Ann. de la Fac. des Sciences de Toulouse, 6 (2):pp 73-96, 1993.

[OT] V. Ovsienko, S. Tabachnikov. Projective differential geometry, old and new. Cambridge tracts in Mathematics, Cambridge University press, 2005.

[Wi] John Williamson. Normal matrices over an arbitrary field of characteristic zero. American Journal of Mathematics, 61:2,pp 335-356, (1939).

Mathematics Department, University of Wisconsin, Madison, Wisconsin 53706

E-mail address: maribeff@math.wisc.edu 